Illinois State University

ISU ReD: Research and eData

Theses and Dissertations

3-11-2016

\title{
Catcalling as a "Double Edged Sword": Midwestern Women, Their Experiences, and the Implications of Men's Catcalling Behaviors
}

Colleen O'Leary

Illinois State University, colleenvoland@gmail.com

Follow this and additional works at: https://ir.library.illinoisstate.edu/etd

Part of the Communication Commons

\section{Recommended Citation}

O'Leary, Colleen, "Catcalling as a "Double Edged Sword": Midwestern Women, Their Experiences, and the Implications of Men's Catcalling Behaviors" (2016). Theses and Dissertations. 535.

https://ir.library.illinoisstate.edu/etd/535

This Thesis is brought to you for free and open access by ISU ReD: Research and eData. It has been accepted for inclusion in Theses and Dissertations by an authorized administrator of ISU ReD: Research and eData. For more information, please contact ISUReD@ilstu.edu. 


\title{
CATCALLING AS A “DOUBLE EDGED SWORD”: MIDWESTERN WOMEN, THEIR EXPERIENCES, AND THE IMPLICATIONS OF MEN'S CATCALLING BEHAVIORS
}

\author{
Colleen O’Leary
}

\section{Pages}

Due to the lack of research surrounding the topic of catcalling, it is essential to give voice to women who have experienced the harm, its effects, and those who perceive it as a societal issue. This study generates discourse regarding Midwestern women's standpoints on catcalling through in-depth respondent interviews about women's experiences and perceptions of catcalling. Thematic analysis of the interviews reveals that catcalling is complex. Women conceptualized catcalling as a primarily negative experience as they associated it with negative emotions and affective states. Women never described catcalling as complimentary, rather, they believed that men perceived their own comments to be compliments. Specifically, the data revealed that women feel that their experiences and perceptions of catcalling are discounted by men and society as catcalling continues to be framed as complimentary.

KEYWORDS: Catcalling, Feminist standpoint theory, Objectification, Emotion. 
CATCALLING AS A "DOUBLE EDGED SWORD”: MIDWESTERN

WOMEN, THEIR EXPERIENCES, AND THE IMPLICATIONS

OF MEN'S CATCALLING BEHAVIORS

COLLEEN O'LEARY

A Thesis Submitted in Partial

Fulfillment of the Requirements

for the Degree of

MASTER OF SCIENCE

School of Communication

ILLINOIS STATE UNIVERSITY

2016 
Copyright 2016 Colleen O’Leary 
CATCALLING AS A “DOUBLE EDGED SWORD”: MIDWESTERN WOMEN, THEIR EXPERIENCES, AND THE IMPLICATIONS OF MEN'S CATCALLING BEHAVIORS

\author{
COLLEEN O'LEARY
}

COMMITTEE MEMBERS:

John R. Baldwin, Chair

Sandra M. Metts

K. Megan Hopper 


\section{ACKNOWLEDGMENTS}

I would like to thank many wonderful people who have helped me throughout this process, as well as my graduate career. First and foremost, I want to extend a special thanks to my committee members, Dr. Sandra Metts and Dr. Megan Hopper, for taking the time to make my thesis the best project it could be. I am grateful for your willingness to provide feedback to help enhance my project. You were both wonderful to work with, and I would not have made it through this process without you. Thank you!

I must send very important recognition to my chair, Dr. John Baldwin. With your assistance, I was able to set high standards for myself and complete a project that I never thought possible when I first came into this program. My thesis would not be half of the project that it is without your help. As a result of your willingness to help whenever I needed assistance, I pushed myself to constantly improve my project. One of the best decisions I made during my graduate career was choosing Dr. Baldwin to be my chair, and I am thankful for your constant support throughout this process.

Next, I want to extend recognition to Patricia Franz for her kindness and selflessness. You always answered my questions, provided me with advice, and extended a helping hand when necessary. I will miss coming to your office and having life chats because you always seemed to make my day a little brighter. Thank you!

To my wonderful colleagues, you never failed to cheer me up or make me laugh when times were tough. Even when I was stressed about assignments and projects, you 
were extremely encouraging, which often restored my confidence, and I felt reassured that I could overcome any challenge I might encounter. Many of you were also willing to pass on invitations to my study to others. If it weren't for you, I would not have completed this project on time. I especially want to thank my mentees for always offering their help when I needed it most. Your kindness and generosity was much appreciated, and I hope you know how much you impacted my second year in this program.

I want to thank all 13 participants for voluntarily participating in my study. These individuals were kind enough to take time out of their days to disclose their experiences, which were often quite personal. I would not have been able to complete this study without your generous participation.

Finally, I wish to thank my friends and family for always reminding me that I can do anything I set my mind to. Your words of encouragement helped me through the highs and lows of my graduate career. You supported me when I wanted to attend graduate school, and I wouldn't have made it through without your encouragement. Thank you for always believing in me!

C.O. 


\section{CONTENTS}

Page

ACKNOWLEDGMENTS $\quad$ i

CONTENTS

CHAPTER

I. STATEMENT OF THE PROBLEM 1

II. REVIEW OF LITERATURE 9

$\begin{array}{ll}\text { Sexual Harassment } & 9\end{array}$

Definitions of Sexual Harassment $\quad 10$

Stranger Harassment 14

Street Harassment 19

$\begin{array}{ll}\text { Catcalling } & 32\end{array}$

Feminist Standpoint Theory $\quad 37$

Summary and Research Questions $\quad 41$

Conclusion 44

III. METHODS 46

Participants 46

Data Collection $\quad 48$

$\begin{array}{ll}\text { Procedure } & 48\end{array}$

Interview Protocol 49

Data Analysis $\quad 51$

Conclusion $\quad 53$

IV. FINDINGS 54

Women's Perceptions of Catcalling $\quad 54$ 
View of Catcalling

Making Connections to the Past 64

$\begin{array}{ll}\text { Affective States } & 66\end{array}$

$\begin{array}{ll}\text { Anger } & 67\end{array}$

Catcaller Induced Face Loss $\quad 69$

Fear: The Desire to Minimize Harm 71

Communicative Responses $\quad 73$

$\begin{array}{ll}\text { Strategies } & 74\end{array}$

Factors that Influence Responses to Catcalling 78

Factors Influencing Perceptions $\quad 81$

Contextual factors $\quad 81$

$\begin{array}{ll}\text { Situational Factors } & 90\end{array}$

Women's Experiences as Trivialized 93

Minimizing Catcalling Behaviors 94

$\begin{array}{ll}\text { Conclusion } & 97\end{array}$

$\begin{array}{ll}\text { V. Discussion } & 99\end{array}$

Discussion of Research Questions 100

"It's not a Compliment but..." 100

"I Felt..." 102

"It's a Lose-Lose for Women" 103

"Halo Effect" 105

"Catcalling Isn't a Serious Issue" 108

Strengths, Limitations, and Directions for Future Research 109

Strengths and Limitations $\quad 109$

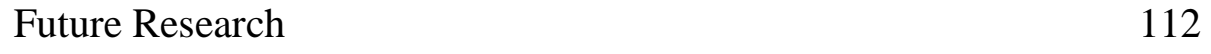

$\begin{array}{ll}\text { Summary } & 116\end{array}$

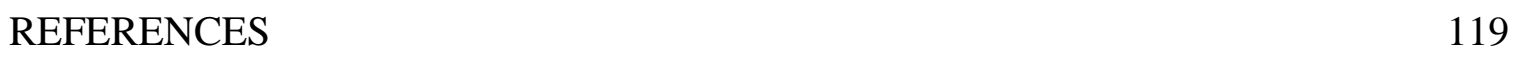

APPENDIX A: Email Message/Facebook Message to Prospective Participants 126 
APPENDIX C: Interview Guide/Question Protocol 


\section{CHAPTER I \\ STATEMENT OF THE PROBLEM}

"My silence does not have anything to do with approval. It is a deep and dark conclusion, accumulating from countless life experiences and enough trial and error: There is no way to fight back and win."

(“Street harassment of women and girls in New York City," 2010).

People encounter various irritations while in public every day. From car honks to construction work, the street can be a source of stress as people try to get to and from particular destinations. While it is common for individuals to experience these minor irritations, there is "also a subcategory of occurrences which affect only part of the population” (Heben, 1994, p. 183). Street harassment is experienced frequently by many women in the United States (Thompson, 1994, p. 314). This widespread practice is often ignored as it is difficult to reprimand harassers. This is due to the fact that harassment tends to occur in passing, making it nearly impossible for victims to identify their harassers. As street harassment continues to be overlooked, millions of women experience a threat to their dignity and safety as they are unable to freely move about a public space without being harassed. Even so, recently, street harassment has become an 
internet sensation as women have taken to the streets to videotape their experiences with harassment. Websites such as Holla Back! and Stop Street Harassment inform the public about instances of catcalling and harassment, which gives women the chance to discuss their own experiences as they are often silenced or deemed insignificant by legislators. This is a result of the unavailability of legal remedies for individuals who are affected by street harassment. In fact, our legal system has "generally refused to acknowledge that street harassment exists" (Heben, 1994, p. 219). Thus, Holla Back! and various other websites have developed campaigns in hopes of combatting street harassment and reshaping laws that fail to reflect women's experiences of street harassment.

Often identified as sexual harassment in public places, street harassment is an experience that is all too common for women. According to Kearl (2010), "Starting at a young age, as many as 80 percent of women around the world face at least occasional unwanted, harassing attention in public places" (p. 3). This can include car honks, winks, and verbal comments. In cases of extreme situations involving street harassment, situations can escalate into sexual assault or even murder. These situations are often documented by the media, which makes it fairly easy for women to observe stories of other women being stalked, catcalled, and street harassed. By viewing these situations through various media outlets, women tend to experience a sense of fear and insecurity when in public. This is not to say that all women feel a sense of fear when alone in public places, but many women feel as if they need to be on guard when a stranger walks by. Representations of women who have fallen victim to street harassment reinforce the belief that "men have control of the streets, and women belong in the home" (Madriz, 
1997, p. 353). Spatial boundaries are thus reinforced, which emphasizes women's inability to leave home without experiencing some form of public scrutiny. As much as websites bring this issue to light, street harassment continues to take place with little repercussions for harassers, which indicates the importance of investigating this topic in hopes of understanding women's experiences and empowering them against street harassment.

Although media outlets tend to expose the destructive consequences of street harassment along with women's disapproval of the behavior, street harassment continues to take place. In October of 2014, two women were violently attacked after responding to their harassers. A young woman was attacked in Queens, NYC on October $1^{\text {st }}$. After refusing to go on a date with her harasser, the man viciously slashed the victim's throat leaving her in critical condition (Feis, 2014). Less than a week later, Mary Spears, a 27year-old woman living in Detroit, was shot and killed after rejecting the romantic advances of her harasser (Lambertz, 2014). These are just two examples of the violence that women could potentially face when responding to catcallers.

Even though street harassment is likely to impact women negatively, little feminist research has been conducted in this area, with it continuing to be identified as a less relevant issue compared to other forms of sexual harassment. Women's experiences of street harassment are normally overlooked because street harassment was not seen as "harassment," until about 20 years ago. Thus, "the conversation has begun about street harassment in public places" but more discussion needs to take place before such 
harassment is viewed as a societal issue deemed punishable by the court system (Laniya, 2005, p. 93).

Men do not often experience street harassment, resulting in their inability to recognize the harmful nature of street harassment. This then reinforces the invisible nature of men's dominance over women and their ability to act upon women without restrictions. Because men are not normally victims of such harassment, they are likely to interpret women's experiences of street harassment as isolated events. In Ellison vs. Brady, the Ninth Circuit Court of Appeals recognized that men tend to view sexual misconduct in a vacuum (Laniya, 2005, p. 104). Rarely is there recognition of harassment as a repeated occurrence with the possibility of the harm escalating into something more dangerous. Perry (2007) argues "that women are expected to submit to an ongoing threat of violence in public spaces because street harassment has been normalized" (p. 126). In fact, because street harassment has become normalized, women tend to devise their own ways to minimize exposure to harassment. Many hesitate to walk alone on the street at night or alter their appearance in order to avoid drawing more attention to themselves (Stanko, 1985, p. 1).

The idea that women should devise their own methods to limit street harassment is also characterized through social narratives such as government documents and police advice. That is, traditionally, magazines emphasizing American etiquette taught women to mute their presence in public spaces by dressing unremarkably, bringing a companion, and adopting distinct behaviors that do not call attention to their presence (Gardner, 1995, p. 19). The canon of psychiatric advice about men warned women that they should not 
assertively react to men who offend them. Voyeurs or offenders were deemed harmless as their whistles were identified as expressions of approval. Women were thus prescribed a set of "right" and "wrong" responses towards offenders. In a 1969 Glamour magazine test titled "Do You Act Like a Beauty?," women were provided with lessons in how to act responsibly in public:

You're walking along the street and a workman whistles appreciatively at you. You:
a. Ignore him.
b. Tell him he's being pretty fresh.
c. Call a cop.
d. Smile in friendly acknowledgment and keep walking.

(Glamour as cited in Gardner, 1995, p. 22-23)

Glamour's correct answer is "d."

Glamour's advice column illustrates that women have been taught for some time that men's verbal comments in regards to a woman's appearance, also known as catcalls, are a means of complimenting a woman. Women are told to accept a man's verbal or nonverbal behavior as complimentary without giving them a choice to interpret the behavior as an inappropriate intrusion into a woman's space. This then trivializes women's experiences of street harassment as women are forced to "bear the burden of protecting themselves from gender-based violence or misogyny" (O’Neil, 2013, p. 13). Men are not held responsible for their actions, which continues to silence women's concern and disapproval of men's continued objectification of women's bodies. 
It is evident that men and even certain women perceive that catcalling is complimentary as men often make evaluative comments about a woman's appearance. This can be problematic as this form of street harassment continues to be reinforced while women's voices are ultimately silenced. Women fail to talk about their experiences, which makes the harm appear to be trivial or even nonexistent. Thus, the ultimate assumption that catcalling involves harmless compliments conceals the pervasive nature of this harm.

Growing up in a sheltered suburban community, I was unaware of the harms that many women face on a daily basis. It was not until my parents gave me permission to watch forms of media that were rated $\mathrm{R}$ or $\mathrm{M}$ for adults, that I was able to observe television shows and movies depicting women who had experienced sexual assault, rape, and street harassment. By observing these harms, I realized at a young age that these harms can be experienced by just about any woman. As a result of being exposed to these forms of media, I became increasingly fearful of experiencing such harms myself. When I was young, I would only walk around my neighborhood at night if I was accompanied by a friend, but if there was a time in which I was alone, I would always look over my shoulder in anticipation of a potential predator.

As an adult, I am still fearful of being attacked by an unknown stranger, and my fear continues to be perpetuated as a result of the forms of catcalling that I often experience. I have watched men leer at me on a daily basis as I walk to class, and I have also experienced verbal insults while walking through cities and suburban streets. These instances have forced me to realize how vulnerable I am at any given place or time. As a 
result of the fear I have developed over time, I am increasingly frustrated and annoyed by catcalls. I feel uncomfortable, angry, and fearful when men proceed to catcall me in public spaces. As such, I find it difficult to comprehend individuals' conceptualizations of catcalling as complimentary. Thus, I sought to discover how women come to understand and evaluate men's catcalling behaviors, as it is possible that women may harbor feelings that are similar or different from my own views of catcalling as some women might enjoy it.

Women face the constant struggle of either risking the possibility of experiencing harassment or altering their normal behaviors of when, where, and how they travel to a desired destination. This indicates the importance of further investigating the topic of catcalling. Within this qualitative study, I conducted in-depth interviews with college aged women in the Midwest from small or mid-sized communities who had experienced men's catcalling behaviors. As most experiences of catcalling take place in large urban areas, the present study is unique as it explored a population that researchers have yet to consider when examining women's experiences of harassment. Specifically, I explored women's emotions and the communicative behaviors enacted when they experience catcalling. Women's emotions have rarely been explored in terms of sexual, stranger, and street harassment. If researchers have examined women's emotions, they have failed to specify the specific emotions women have felt. As a result, the current study contributed to this lack of research as women's emotions play a vital role when it comes to experiences of catcalling. It is also necessary to bring about awareness in regards to women's conceptualizations of catcalling behaviors as complimentary, harassing, or a 
combination of both. If women condone catcalling behaviors, it is possible that women have catcalled or have contemplated catcalling men as well.

Primarily, I hope to give a voice to women who have been affected by catcalling in order to shed light on their experiences as these are often internalized rather than communicated with others. With my research, I hope to provide scholars with new insight on a topic that has received little scholarly attention, especially within the realm of communication. More specifically, researchers within the fields of interpersonal, feminist, and women and gender studies may benefit from the current study. As a whole, catcalling has received a great deal of attention in the media but little scholarly attention. My goal is to provide scholars with a basis for future studies that focus on experiences of harassment. While many women may view men's catcalling behavior as complimentary, researchers are unable to come to such a conclusion without gathering women's personal standpoints on catcalling. 


\section{CHAPTER II}

\section{REVIEW OF LITERATURE}

The previous chapter discussed street harassment, women as victims of such harassment, and the harms it often causes. The examination of a subset of street harassment, catcalling, is crucial for interpersonal, feminist, and women and gender studies research as little communication scholarship has examined women's standpoints on catcalling. In this chapter, I discuss previous research pertaining to forms of harassment, specifically sexual, stranger, and street harassment. I will also examine previous research focused on catcalling, a subcategory of street harassment, as well as the theoretical framework of Feminist Standpoint Theory.

\section{Sexual Harassment}

Over the last 20 years, researchers have determined that sexual harassment occurs in many different countries and cultures. In the United States, sexual harassment is generally defined using gender neutral verbiage, but most research indicates that women are most often victims of sexual harassment while men are the perpetrators (Pryor \& Meyers, 2000, p. 219). The sexual harassment of women is widespread and has been found to negatively impact women's productivity in the workplace as well as women's stability (Celik \& Celik, 2007; Pryor, 1998). Even though sexual harassment has become a vital issue in terms of social and political importance, 50 years ago, the topic of sexual 
harassment was under-researched as scholars had yet to name the harm. Now that sexual harassment has been named and defined, it is recognized as a valid harm experienced by men and women (Laniya, 2005, p. 92). Although great strides have been made towards condemning sexual harassment as in the development of "Title VII and state nondiscrimination laws," sexual harassment is still largely referred to in relation to the workplace (p. 92). Thus, definitions of sexual harassment originated with considerations of workplace harassment, though recent research looks at the factors leading to harassment much more broadly.

\section{Definitions of Sexual Harassment}

Sexual harassment is one of the most aggressive forms of harassment executed in the workplace (Berdahl, 2007, p. 427). It is harmful on an individual as well as a social level as it often involves unwanted and undesired sexual advances. Researchers have found that sexual harassment not only involves potential job loss, but it tends to have debilitating psychological impacts on an individual's health and well-being (Mackinnon, 1979, p. 160). Historically, research on sexual harassment focused on contexts such as the workplace or schools, in which the perpetrator of the harassment is known by the victim. These contexts became the prime focus of research during the 1980s as discrimination was prevalent and in need of eradication. Aside from "its importance as social policy," the elimination of sexual harassment took on a critical role in legal areas (Hickson, Grierson, \& Linder, 1990, p. 22). In 1984, the amount of federal cases regarding sexual harassment was at an all-time high, with 6,342 cases. 
Because sexual harassment was, and continues to be a prevalent and complex issue, researchers developed multiple definitions of the concept. Hornstein (1984) defines sexual harassment as the "repeated demands or continuing behavior of supervisors or coworkers that add a discriminating condition or term of employment or create a harmful work environment for women" (p. 233). Other definitions utilize the concept of sexual coercion, which Spitzberg (1998) identifies as "the expressed dispreferred inducement of sexual relations through force, threat of force, deceit, or the practical removal of choice from the victim" (p. 189). Women are often coerced into performing sexual acts or submitting to sexual advances as a condition of employment. Sexual harassment has also been defined within the legal sphere. One type of harassment, Quid pro quo harassment, involves the loss or denial of a job-related benefit, such as a promotion or salary raise, as a result of refusing to cooperate with sexual advances (Berdahl, 2007). This type of harassment normally involves a male boss harassing a female subordinate. When this type of harassment was initially defined in the 1970s, courts focused on the harassers' motivations. They assumed that harassers were motivated by sexual desire towards their targets until researchers discovered other motivations for sexual harassment. Thus, it can be determined that, historically, researchers have focused on the relationship between the perpetrator and the victim by means of quid pro quo harassment in the workplace.

A more holistic approach to sexual harassment adds to quid pro quo harassment two other possible forms: gender harassment, and unwanted sexual attention (Gelfand, Fitzgerald, \& Drasgow, 1995, p. 167). Gelfland et al. analyzed female university students' self-reported experiences of sexual harassment and determined that both gender 
harassment and unwanted sexual attention affect women's experiences in the workplace. Gender harassment refers to "the degradation of a gender group via jokes or other offensive stimuli," while unwanted sexual attention involves "the degradation of an individual via inappropriate communications or sexually-related behavior" (Wesselman \& Kelly, 2010, p. 451).

Men and women seem to see harassment differently. Blumenthal (1998) conducted a meta-analytic review of 111 studies that focused on gender difference in relation to perceptions of sexual harassment. Blumenthal found that women identify a broader range of behaviors as sexual harassment compared to men. Rotundo, Nguyen, and Sackett (2001) extended this study and determined that females were more likely to label social-sexual behaviors "that reflect derogatory attitudes and involve dating pressure or physical sexual contact" as sexual harassment compared to males (p. 920). Thus, it can be determined that men and women view sexual harassment differently, which can have implications. Men might engage in behaviors that they do not believe constitutes sexual harassment, which could result in legal punishment as well as create an environment that women perceive as unsafe.

Factors contributing to sexual harassment. Researchers have determined culture to be a contributing factor of harassment. That is, organizational culture plays an important role in the definition and understanding of sexual harassment. Sexual harassment practices such as policies and reporting procedures contribute to an employee's overall understanding of what constitutes sexual harassment. Thus, organizations' responses to issues involving sexual harassment create "a social system in 
which employees make sense of the working environment" (Keyton, Ferguson, \& Rhodes, 2001, p. 35). This environment guides employees' behavior as well as their evaluation of others' behavior. Whether an organization tolerates or prohibits sexual harassment influences an employee's perceptions and behaviors, which, in turn reproduces conditions that foster or suppress harassment. For example, Firestone and Harris (1999) determined that military cultures are more conducive to sexual harassment than other organizational cultures because they are aggressive and masculine in nature. Organizations with highly masculine environments tend to tolerate sexual harassment, which is likely to influence employees' perceptions of sexual harassment.

Many situational factors also influence men's likelihood to sexually harass. For example, Pryor, LaVite, and Stoller (1993) determined that perceptions of group norms predict sexual harassment. Group norms are understood as consensual standards that deem a behavior appropriate in a specific context. Pryor et al. argue that the composition of a group influences men's likelihood to sexually harass. That is, if group members perceive the norms to be more accepting of sexual harassment, members are more inclined to engage in sexually harassing behaviors. The presence of other group members also contributes to the likelihood of men engaging in sexually harassing behaviors.

In addition, behaviors tend to be more aggressive and less socially regulated when the source of the behavior is unknown. Postmes and Spears (1998) suggest that individuals who are in situations that offer greater anonymity are more likely to adhere to group norms. Research by Pryor (1998) indicates that an essential element in the Likelihood to Sexually Harass (LSH) scale is respondents' belief that their sexually 
harassing tendencies are anonymous. Therefore, group norms that are more tolerant of sexually harassing behaviors and situations high in anonymity tend to foster an environment where an individual is likely to engage in such behaviors. The special impact of anonymity on harassing behaviors and on those who are harassed has led to a specific study of sexual harassment—stranger harassment.

\section{Stranger Harassment}

Stranger harassment versus sexual harassment. Stranger harassment is a form of sexual harassment that occurs in public places and is perpetrated by a harasser who is unknown to the victim (Bowman 1993; Fairchild \& Rudman 2008). It is so pervasive that it is deemed a part of the social fabric of public life (Gardner, 1995). Gardner (1995) argues that stranger harassment is unique because it is conducted via methods of communication in public spaces and includes "verbal and nonverbal behavior, such as wolf-whistles, leers, winks, grabs, pinches, catcalls, and street remarks; the remarks are frequently sexual in nature and comment evaluatively on a woman's physical appearance or on her presence in public" (Bowman, 1993, p. 523).

Macmillan, Nierobisz, and Welsh (2000) provide one of the first examinations of stranger harassment. The researchers analyzed data from the 1993 Violence Against Women Survey (VAWS), which included a national sample of 12,300 Canadian Women. Macmillan and colleagues found that more than 80 percent of the women had experienced some form of stranger harassment, and 30 percent had experienced confrontational forms of harassment. They also found that stranger harassment affected women's perceptions of safety. Women had an increased fear of "walking alone at night, 
using public transportation, and walking alone in a parking garage" (p. 319). This demonstrates that the more that women experience stranger harassment, the less safe they feel in public spaces. As a result, women develop an increased fear of victimization, which stems from the combination of sexualized interactions and an unknown harasser.

Researchers have also examined the behaviors of known versus stranger harassers in terms of the way these behaviors affect women differently. A study of harassment from coworkers (sexual harassment) versus strangers (stranger harassment) revealed that behaviors perpetrated by stranger harassers lead to increased ratings of severity and negative emotions (McCarty, Iannone, Kelly, 2014). When it comes to known harassers, as with sexual harassment, the victim has access to information about the harasser such as how consistent and distinct the harassment is. The harasser is thus granted more leeway than a stranger who engages in the same behavior. Thus, the behavior of a known harasser is interpreted as less threatening, which typically results in less negative reactions and outcomes for the victim (McCarty et al., 2014). These findings demonstrate that stranger harassment is a more pervasive harm than sexual harassment as it leads to considerably negative consequences for women.

Stranger harassment is not only perpetrated by individual harassers, but also by groups of harassers. Wesselmann and Kelly (2010) conducted the first empirical study that examined different personality and social factors that motivate college males to engage in stranger harassment. Findings revealed that college men are more likely to engage in stranger harassment when in a group rather than alone (p. 454). In addition, group contexts serve as a means for group bonding as men align their behavior with other 
men in the group in order to facilitate a group bond (Hunt \& Gonsalkorale, 2014, p. 16). It tends to strengthen "the cohesion of the male in-group at the expense of the female outgroup" (Thomae \& Pina, 2015, p. 188). This coincides with sexist humor, which serves as a form of male-bonding. Lyman (1987) examined the male bonds that are often created in college fraternities. He argued that sexist jokes and taunts serve as a form of group bonding in fraternity life. The men in his study felt as if directing anger and aggression towards women was a behavior that they were allowed to execute because social norms permitted it. Disparagement humor that targets an out-group such as women increases in-group morale, while simultaneously developing hostile attitudes towards the out-group (Hunt \& Gonsalkorale, 2014). In sum, stranger harassment involves aspects of male bonding as men are more likely to engage in stranger harassment in a group setting where social norms encourage it.

Although stranger harassment often takes place in public places, it is worth noting that in today's society, stranger harassment can also occur online. Given the rapid growth in communication technologies and technology use, stranger harassment is likely to be more frequent online than face-to-face. Megarry (2014) argues that male voices carry more "authoritative weight than female voices" on social media sites such as Twitter. As a result, women continue to be disproportionately targeted for harassment by means of messages involving graphic descriptions of violence as well as threats of death. Megarry examined the pertinence of such harassment by looking at a specific Twitter hashtag, \#mencallmethings, which women utilize to discuss the online harassment that they receive from men. She considered tweets from November 7, 2011, when the hashtag was 
first created, to December 7, 2011. Analyzing isolated tweets of women's examples of online harassment revealed that women frequently experienced threatening abuse online (Megarry, 2014). Women would receive comments "on their physical attributes," which were made "in a violent and aggressive manner" (p. 50). Comments involved name calling, threats of violence, and a preoccupation with women's physical appearance. The results of this study indicated that women's experiences of online abuse are more common than one would think, which was also determined by Laura Bates, founder of the Everyday Sexism Project and the Twitter feed @everydaysexism. An examination of her Twitter feed revealed that Bates was exposed to an immense amount of hate-filled messages that ranged from "graphic descriptions of domestic violence to threats of torture, death, and rape" (Bates, 2013). Thus, women are unable to avoid threatening abuse from men, as they not only experience violent behaviors from strangers in public places, but also online via social media sites such as Twitter.

Contextual influences. Although previous research has demonstrated that stranger harassment negatively impacts women, scholars have suggested that such harassment is not loathed by every woman (Fairchild, 2010, p. 192). Viewing stranger harassment as a compliment is a coping mechanism that some women utilize in order to mitigate the negative effects that stranger harassment tends to have on a woman's sense of safety and well-being. Fairchild (2010) demonstrates that women are almost socially conditioned to view men's behavior as complimentary, or at the very least, as inoffensive. Evidence also suggests that the same woman might enjoy a compliment by a stranger one day, and be infuriated by a different compliment the next. 
Fairchild (2010) utilized hypothetical vignettes to examine what "contextual effects influence the perception of stranger harassment" (p. 192). Participants received one of eleven vignettes asking participants to take the perspective of a target of stranger harassment and indicate how they would feel. Fairchild predicted that the context in which a harassing behavior occurs is likely to alter the perception of the target. Harassing behaviors might be viewed as compliments in one situation but threats in a different situation. Fairchild discovered that various factors such as time of day, location, and whether the woman was alone or with friends were related to women's perceptions of the situation as more frightening or enjoyable. Age and attractiveness of the perpetrator also elicited particular emotions for the women. That is, a younger, more attractive harasser elicited less negative emotions than the condition with the older harasser at night. Even though women experienced less negative emotions as a result of certain contextual influences, across all conditions, women were equally fearful of the harassing behaviors and utilized passive coping strategies such as ignoring the harasser (p. 210). Even if a woman views stranger harassment as a compliment, it is likely that she will experience negative emotions and a need to engage in coping strategies in order to manage the negative repercussions that result from experiencing stranger harassment.

Even though stranger harassment has been examined in various studies, the harm is "ignored in the social science and feminist literature" (Fairchild \& Rudman, 2008, p. 339). Stranger harassment tends to be overlooked because it is enacted by a stranger who disappears quickly after the harassment takes place (Bowman, 1993; Nielsen, 2000). This makes it difficult for victims to identify harassers, making it unlikely for a law to be 
created, much less supported, to punish harassers. There are very few policies that protect victims of stranger harassment in public spaces as such laws are difficult to enforce. On the other hand, sexual harassment policies have been implemented to protect women in workplace and academic settings. The fact that stranger harassment is often overlooked by researchers and difficult to reprimand signifies the need for researchers to continue examining it as a unique form of harassment. Incidents of stranger harassment are not irrelevant as they are deeply felt and important to individuals who are victims of such harassment (Gardner, 1995, p. 14). Ignoring stranger harassment deprives those who suffer the harm, those who perpetuate it, and the researchers who study it, of an understanding of the public realm that most of us find difficult to avoid.

\section{Street Harassment}

Street harassment is a particular form of stranger harassment that involves both verbal and nonverbal behaviors in street contexts. The actions that comprise street harassment are viewed as solitary forms of harassment, as opposed to sexual harassment in general, and its occurrence specifically on the street, as opposed to other contexts or on the Internet, make it distinct from the more general stranger harassment. Like other forms of harassment, street harassment is associated with specific effects and factors.

The uniqueness of street harassment. Micaela di Leonardo (1981) identifies specific characteristics that distinguish street harassment from other forms of harassment. Street harassment occurs when a male stranger intrudes on a woman's attention by using words, looks, or physical contact, which essentially defines a woman as a sexual object 
(Leonardo, 1981, p. 51). Researchers have also recognized particular characteristics of street harassment that perpetuate its identification as a gender-based harm:

(1) the targets of street harassment are female; (2) the harassers are male; (3) the harassers are unacquainted with their targets; (4) the encounter is face to face; (5) the forum is a public one, such as a street, sidewalk, bus, bus station, taxi, or other place to which the public generally has access; but (6) the content of the speech, if any, is not intended as public discourse. Rather, the remarks are aimed at the individual (although the harasser may intend that they be overheard by comrades or passers-by), and they are objectively degrading, objectifying, humiliating, and frequently threatening in nature. (Bowman, 1993, p. 524).

Street harassment is often deemed a subcategory of sexual harassment because it involves unwanted sexual acts that are deliberately perpetrated by a harasser. Even though street harassment and sexual harassment involve undesired sexual acts, there are several distinctions between the types of harassment, the first being locale. Sexual harassment often occurs in the absence of an audience such as the workplace, educational institutions, and in the home, while street harassment occurs in public spaces such as the street (Smith, 2008, p. 3). Also, both forms of harassment are comprised of different behaviors. Sexual harassment tends to involve physical contact and verbal remarks, while street harassment often begins with a man leering at his victim or gazing uncomfortably at her. It may then move to more threatening behaviors involving unsolicited sexual comments and may end with physical contact such as unwanted grabs, pinching, or groping (Chhun, 2011, p. 277). Thus, sexual harassment is not very easy to conceal 
because the harasser is normally an acquaintance who engages in physical contact with the victim. This makes it much easier to convict those who sexually harass.

Another difference is that laws have been established that sanction acts of sexual harassment, but the same cannot be said for street harassment. Even though street harassment has come to be identified as a means of subordination, it "has not generally been viewed by academics, judges, or legislators as a problem requiring legal redress" ( $\mathrm{p}$. 519). For example, the identification of sexual harassment as a reprehensible harm in the late 1960s, early 1970s, made the issue of street harassment seem trivial. During that period of time, sexual harassment was named and defined in the eyes of the law. It was identified as a form of sex discrimination and became forbidden under the Civil Rights Act of 1964. As a result, people began to identify street harassment as a harmless, "boyswill-be-boys" type of behavior that bothered many women for supposedly fictitious reasons (Davis, 1994, p.152).

The supposed harmless and public nature of street harassment links to its prevalence. Benard and Schlaffer (1984) were two of the first scholars to investigate the notion of street harassment. They conducted a study in Vienna and found that women were being harassed regardless of their age or level of attractiveness. Gardner (1995) found the same to be true in the United States. She found that men on the streets of Indianapolis were likely to harass women irrespective of the women's race and socioeconomic status. Men were also of different races and socioeconomic backgrounds, demonstrating that the behavior is unrelated to demographic-type categories. Gardner (1995) defined street harassment as "that group of abuse, harryings, and annoyances 
characteristic of public places and uniquely facilitated by communication in public, including pinching, slapping, hitting, shouted remarks, vulgarity, insults, ogling, and stalking" (p. 4). Over a five-year time span, she made observations of a field setting and conducted 300 in-depth interviews of women. From this research, Gardner concluded that women experience various forms of harassment ranging from insults to grabs by strange men in public places. Participants were sure to emphasize that "no one-not the perpetrator and probably no official—will think anything of note has happened" (p. 4). As displayed, victims often identify street harassment negatively, as a means in which a man asserts sexual power over a woman through an undesired intrusion into a woman's personal space. Although this is true, as with stranger harassment generally, victims might also evaluate street harassing behaviors positively, negatively, or a combination of both. Various factors such as level of attractiveness of harassers and group norms are likely to influence victims' evaluations of street harassing behaviors as complimentary or offensive.

Factors influencing street harassing behaviors. Lack of criminalization is one of several factors that might impact incidences and forms of street harassment. Street harassment often takes place in large urban areas such as Washington D.C., Chicago, and New York City. The only places where street harassment is not common, as identified in Benard and Schlaffer's (1984) empirical study, are small villages, residential suburban areas, and regimes in which women are veiled and prohibited from moving about in public spaces. These researchers concluded that street harassment is confined to the "genuinely public world" where individuals are unacquainted with one another (p. 398). 
They also contend that harassment occurs in the street because societies have historically utilized streets as markers of order and status. Historically, on the streets, members of subordinate groups were expected to "salute, take off their hat, or jump down from the sidewalk to make way for the members of the superior group" (p. 398). Although researchers have identified urban areas as the most common locations in which harassment takes place, these studies are somewhat outdated. It is possible that harassment does take place in contexts such as suburban areas, but more research is needed for scholars to make this claim. The present study will contribute to this lack of research by exploring Midwestern women's experiences of a specific form of harassment, catcalling, which will likely take place outside of urban areas.

Individuals' behaviors and appearances in public spaces vary from behaviors in private settings. That is, communication in public spaces is appearance dependent. Individuals rely on a stranger's visible form in order to judge the stranger's identity with the understanding that strangers will judge them in the same manner. Communication within this specific context relies on the appraisal of an individual's physical characteristics, nonverbal communication, and dress (Gardner, 1995, p. 52). Because public places, such as streets, are appearance dependent, men tend to believe that women dress a certain way in hopes of receiving a comment that evaluates their appearance. This belief may influence the continued enactment of street harassing behaviors. Kissling and Kramarae (1991) conducted an analysis of a discussion of street remarks. The discussion took place in a notes file titled "Sexnotes" located on a computer-based research system. 
The system was utilized by students and faculty at large Midwestern University. One man discussed how clothing acts as a symbol system:

How you dress is inevitably going to send messages to the people you encounter ....Whores don't dress like whores just for the hell of it. They dress like whores because it's enticing and inviting.... There are clothes that say it without words. (p. 81)

This implies that men perceive women to dress in a particular fashion in hopes of receiving evaluative comments. The man's comment also points to the assumption that women access public spaces in order to entertain and appease men (Davis, 1994, p. 151). It is, in fact, unlikely that women dress to attract the negative attention that constitutes street harassment. In addition, as noted above, women are harassed regardless of the clothes that they wear. Physical characteristics might contribute to men's enactment of street remarks, but scholars argue that street harassment is "a universalizing experience one that virtually all women share" (Bowman, 1993, p. 534). Thus, those who claim that women are responsible for their own sexual objectification are blaming the victim rather than identifying the problem at hand, the harasser and his actions.

Women are often the victims of such intrusions as a result of the asymmetrical rules of conduct that guide men and women's behavior in public spaces (Kissling \& Kramarae, 1991, p. 75). The concept of civil inattention described by Goffman (1963) emphasizes that strangers behave in a specific manner in public spaces by ignoring one another with the exception of brief eye contact. Two exceptions to this behavior are when a similarity exists between the strangers or when a stranger is viewed as an open person 
(Lord, 2009, p. 2). Lord (2009) identifies an open person as someone "who falls into a category that allows civil inattention to lapse" such as individuals who do not meet society's standards of an ideal person (p. 2). Men have essentially learned to treat women as open persons while in public places. That is, breaches of civil inattention are deemed acceptable forms of male behavior. It is evident that men utilize these breaches as a means of asserting their right to comment on a woman's body or appearance, thus defining women as objects and men as subjects who hold power over women (Tuerkheimer, 1997, p. 186).

The context in which street harassment occurs also causes women to react in a particular manner. Women often reply to their harassers with a "thank you." As Davis (1994) illustrates in her account of the normative characteristics of street harassment, replying with a comment is an unacceptable response. When a woman uses "thank you" in response to a harasser, it "creates a dialogue which usually requires two subjects" ( $\mathrm{p}$. 139). Women are thus placing themselves in the subject position, which frustrates harassers due to their intent to objectify women and place them in the "other" position. The idea that a typical response to a compliment, "thank you," frustrates harassers, calls into question men's identification of street harassment as complimentary.

Gardner (1980) argues that street remarks violate the norms of complimentary behavior. That is, a proper compliment is not given by a stranger in a public place, especially strangers of the opposite sex. Compliment etiquette also indicates that the proper response to a compliment is "thank you" (Gardner, 1980, p. 340). The fact that a woman who responds with a "thank you" tends to escalate street harassment into abuse 
signifies that street remarks violate compliment etiquette. Street remarks are actually far from complimentary as they "often accuse women of inferior looks, improper carriage or attire, inappropriate actions, and moral defects" (p. 333). Even though researchers have established that street remarks violate norms of complimentary behavior, men continue to street harass, which signifies the need to examine other factors that influence men's enactment of street harassment.

Along with men's conceptualization of street harassment as complimentary, the likelihood of bystander intervention is another potential factor that might influence harassing behaviors. This is identified as the bystander effect as individuals experience conflicting thoughts in regards to intervening. Individuals are less likely to intervene in contexts such as residential areas or streets as such contexts encourage bystanders "to resolve the conflict in the direction of nonintervention" (Darley \& Latané, 1968, p. 377). In street scenarios, it is highly unlikely that bystanders will intervene when men engage in harassing behaviors as a result of the belief that someone else will assist the victim. Because others are present, the cost of not responding is drastically reduced, which leads bystanders to diffuse responsibility among other observers. Thus, people are essentially "more likely to receive help when a single bystander is present, than when a group of bystanders are present" (Levine, 1999, p. 3). Because women experience street harassment in the presence of strangers, the ambiguous nature of the relationship between the victim and the bystander decreases the likelihood of bystander intervention. Thus, social norms in street contexts discourage intervention as it would be inappropriate for an individual to disrupt normative patterns by addressing such harassment. 
Female victims also tend to believe that laws prohibiting street harassment might burden the courts as officials would be punishing victims right and left. Because female victims avoid favoring laws that they think would be impossible to enforce, men continue to engage in street harassing behaviors (Nielson, 2000, p. 1082). When the law comes into play, street harassment is viewed as a minor offense compared to other forms of harassment such as race-based discrimination and sexual assault. Nielsen determined that the perceived legality of such speech is observed as the primary mechanism through which victims shift their understanding of the severity of street harassment. Particularly troubling experiences involving street harassment transform into something that should be tolerated. This was particularly evident when Nielsen interviewed 100 participants about legal consciousness in relation to street harassment. Female participants were likely to identify the uncomfortable and unpleasant nature of these experiences. Even so, they were more likely to cite the impracticality of the legal regulation of such behaviors. That is, experiencing "these harms does not translate into supporting its legal regulation" (p. 1056). Victims believe that legal regulation cannot really help as it is difficult to identify perpetrators, and street harassment is often considered a minor offense compared to other more serious offenses. As long "as there are more serious offenses occurring that demand official attention," many people feel that street harassment will have to wait (p. 1083).

Effects of street harassment. Street harassment tends to harm women physically and mentally. Women experience physical reactions to street harassment such as "muscle tension, stopped breathing, numbness, dizziness, nausea, constriction of the throat, trembling, rise in bile in the throat and pounding heart" (as cited in Tran, 2015, p. 187). 
Street harassment incidents can escalate into life-threatening instances. A woman who either chooses to ignore or confront her harasser risks angering him. For example, in January of 2013, a woman was walking through a San Francisco neighborhood when she was approached by a man making sexual comments towards her (Kearl, 2010). The woman responded to the man by rejecting him, and as a result, the man proceeded to slash her face as well as stab her in the arm.

Street harassment can also cause mental and emotional harm as victims tend to feel angry, fearful, and humiliated. Tuerkheimer (1997) emphasizes that street harassment inflicts a deep harm on victims even if a woman's body remains physically untouched (p. 190). Women are thus psychologically oppressed as a result of street harassment. Bartky (1990) identifies psychological oppression as being "weighed down in your mind; it is to have a harsh dominion exercised over your self-esteem” (p. 22). Bowman (1993) connects the topic of psychological oppression to that of street harassment in terms of women's reaction to the harm:

Women who are harassed on the street typically do not respond to the harasser but instead try to ignore him, or, more accurately, pretend to ignore him.... They freeze; they put on a blank face; they try to pretend that nothing is happening. When women take these evasive actions in an effort to mask feelings of invasion, anger, humiliation, and fear, they suffer a psychological beating in the form of emotional distress and feelings of disempowerment. (p. 537)

Thus, passively ignoring the problem reinforces the structures of power in society that fail to acknowledge street harassment as harmful. Whether or not a woman remains 
silent, she is likely to "emerge from the encounter with an overwhelming sense of disempowerment" (Tuerkheimer, 1997, p. 191). This vicious cycle continues to psychologically oppress women while simultaneously defining them as sexual objects.

By failing to recognize street harassment as a social problem, society reinforces the objectification of women's bodies. Objectification theory asserts that women are "treated as a body (or collection of body parts) valued for its use to (or consumption by others)" (Fredrickson \& Roberts, 1997, p. 174). Bodies thus exist within cultural contexts, and hence are constructed through sociocultural discourses. Objectification is a key component of street harassment as women are verbally harassed about their looks, which makes them nothing more than mere objects present for men's satisfaction. Because street harassment classifies women as sexual parts, women are unable to represent themselves as individuals. Women are not only objectified via street harassment, but women's bodies are relentlessly "regarded as sexual objects through pornography, mass media, and advertising" (Fairchild, 2008, p. 342). Through these media, women have been portrayed as objects with the possibility of being gazed upon by men (Young, 2005, p. 44). As a result, men begin to view women solely as sexual parts. According to Fredrickson and Roberts (1997), the consequences of sexual objectification arise when a woman begins to self-objectify. In doing so, women begin to think of themselves as sex objects, which leads to negative self-evaluations resulting in depression and anxiety. Thus, street harassment promotes "psychological and behavioral problems in women through its link to self-objectification" (Fairchild, 2008, p. 353). Street harassment causes women to feel self-conscious about their appearance because 
they are constantly reminded that their bodies are being observed. Thus, it can be determined that objectification and mental health both play a role in street harassment as they tend to have negative effects on women.

Street harassment not only affects women physically and mentally, it also hinders women's mobility by infringing on their ability to access the street. Free access is often denied to women, which forces them to change the routes that they travel while also avoiding streets at night (Laniya, 2005, p. 107). This limitation relegates women to the private sphere of the home, which Bowman (1993) calls the "informal ghettoization of women" (p. 520). Urban environments become uncomfortable and hostile as women are constantly reminded that they are not welcome in the public sphere. Street harassment thus implies "either that women are acting out of role simply by their presence in public or that a part of their role is in fact to be open to the public" (p. 526). Bowman notes that street harassment essentially offsets the gains that women have made in other spheres such as employment and politics (p. 539). Women are seemingly limited in "simpler choices - whether to go to the movies alone, where to walk or jog, whether to answer the door or telephone" (p. 539).

Moreover, street harassment produces an environment of sexual terrorism. Sheffield (1987) defines sexual terrorism as a system by which males frighten and, through fear, control and dominate females. Sexual terrorism is essentially manifested through both implied and actual performances of violence such as rape and workplace sexual harassment. Sheffield (1989) emphasizes that "all females are potential victims at any age, any time, or in any place” to harms such as street harassment (p. 483). 
Kissling (1991) points to street harassment as a factor in creating a sexually terroristic culture (p. 456). Street harassment causes women to feel a sense of vulnerability, which reinforces fear of rape along with other acts of terrorism. Sexual terrorism appropriately describes street harassment because women believe that it will occur, but are often unsure when or how it will occur.

Sexual terrorism and the fear of rape combine the physical and emotional impacts of street harassment noted above. Whenever "a strange man addresses a woman on the street, she must entertain the possibility that he might rape her" (Bowman, 1993, p. 517). This fear is realistic as rape can begin with the act of street harassment (Davis, 1994, p. 140). Potential rapists may test a victim's accessibility by utilizing forms of street harassment in order to determine which woman is an easy target, with an ability to be intimidated. This is often identified as rape-testing because potential rapists test their victims by making sexualized remarks (Bowman, 1993, p. 517). If the target reacts in a passive manner, the rapist is more likely to rape the target.

Thus, it can be determined that "the connection between rape and harassment is not just in the mind of the woman" (Bowman, 1993, p. 517). Street harassment can function as a precursor to rape as it creates a sexually terroristic environment that women are unable to escape. When women experience street harassment, they are reminded of their subordination and the fact that they are always vulnerable to forms of sexual violence (Tuerkheimer, 1997, p. 187). Even though street harassment can function as a precursor to rape, men often fail to recognize that street harassment is essentially a tool of sexual terrorism. The silencing of women's experiences of street harassment inherently 
supports the system of sexual terrorism. In order to understand the harms that women face in street contexts, it is necessary to recognize the role that street harassment plays within the larger sexually terroristic environment.

\section{Catcalling}

Women are often affected by catcalling as $61 \%$ of female participants reported experiencing catcalls every day (Nielsen, 2000, p. 1067). This demonstrates the prevalent nature of catcalling and the need for researchers to continue examining this phenomenon (Rawlins, 2012, p. 489). The term "catcalling" is often discussed in relation to street harassment and stranger harassment as catcalling is comprised of elements from both forms of harassment. Researchers identify catcalling and street harassment as subsets of the larger domains of stranger and sexual harassment. That being said, it is necessary to identify the similarities and differences between these constructs.

Chhun (2011) identifies catcalling as the "use of crude language, verbal expression, and nonverbal expression that takes place in public areas such as streets, sidewalks, or bus stops" (p. 276). Verbal expressions of catcalling tend to involve wolfwhistles or comments that evaluate a woman's appearance. Nonverbal expressions often include leers as well as physical gestures that act as a means to rate a woman's physical appearance (p. 276). In terms of locale, catcalling coincides with the concept of stranger harassment because it takes place in public areas as most private areas have strict laws that prohibit verbal expressions such as sexual harassment in the workplace. It is also important to note that in the United States, legal remedies do not exist to reprimand 
catcallers. This stems from the fact that catcalling does not involve physical contact in which harassment includes, as noted above.

Street harassment can present the threat of physical danger as the harasser physically violates a woman's right to privacy by invading her space or obstructing her path. On the other hand, catcalling rarely involves the potential of physical danger. A catcall may be made from the inside of a car, from the second floor of a building, or from the other side of a fence. In these cases, catcallers keep their distance, unlike most street harassers who force themselves into a woman's personal space. As street harassment often involves stalking and physical harm, it is possible for such acts to be reprimanded. Unlike street harassment, catcalling normally involves verbal comments, which contributes to law officials' inability to regulate catcalling. Any attempt to create a legal remedy for women who are catcalled in public spaces "gives rise to many arguments concerning the First Amendment and the protection of free speech rights" (Heben, 1994, p.184). Because individuals celebrate the notion of free speech as a canon of American democracy, law officials are wary about placing restrictions on catcalling. Even so, Nielsen (2000) asserts that catcalling should be considered offensive public speech as it involves degrading remarks that reinforce existing hierarchies of gender. As courts have yet to recognize catcalling as offensive speech, it continues to remain invisible within the legal realm, which reinforces the notion of catcalling as a trivial experience.

Another element of catcalling is that of forced communication in which a catcaller symbolically forces himself into a woman's space by means of verbal expression (Chhun, 2011, p. 277). As a result, women's behaviors are restricted as they feel unable to move 
about a public space without experiencing some form of catcalling. This connects to the concept of street harassment because harassers invade a woman's physical space via nonverbal actions. Catcalling, like street harassment, restricts women's movements in public spaces while simultaneously objectifying, degrading, and subordinating them.

Little research has investigated catcalling as a separate form of harassment especially in terms of the effects of catcalling on women's well-being. Studies of sexual harassment as well as street harassment have shown that victims suffer from emotional distress, which tends to be accompanied by anxiety, depression, and disgust (Bowman, 1993, p. 538). Catcalling is likely to trigger harms such as psychological trauma and emotions such as fear because women who are publicly insulted "experience a psychological toll from feelings of degradation, embarrassment, and helplessness in addition to feelings of shame about their bodies" (Chhun, 2011, p. 290).

At the same time, not all catcalling may involve insulting comments; rather, it might even be framed as complimentary, for example, highlighting a woman's beautiful appearance. Thus, another manner in which catcalling coincides with the concept of street harassment is that catcalling sexually objectifies women, which leads to women's selfobjectification. As a result of being reduced to the status of a mere object, a woman begins to adopt an observer perspective viewing and treating herself as an object (Fredrickson \& Roberts, 1997). In an interview with CNN, Kimberly Fairchild emphasizes that there is evidence indicating that the sexually objectifying nature of catcalling leads women to self-objectify, taking more of an emotional toll on women than they realize. She indicates that catcalling "encourages women to look at themselves as 
body parts instead of as full, whole, intelligent human beings" (Grossman, 2008, p. 1). Catcalling — even that which is supposedly complimentary—sexually objectifies women, possibly leading women to objectify themselves as in the way street harassment causes women to self-objectify.

Even though women tend to suffer as a result of men's catcalling behaviors, their experiences are often silenced. This stems from men's inability to understand the impact catcalling has on women. That is, "some men believe the purpose of the verbal act is to compliment" and that reversing gender roles would demonstrate men's appreciation of catcalling (Chhun, 2011, p. 280). An example of the reversal of gender roles emphasizes that being objectified evokes feelings of anger as well as humiliation. Thompson (1994) reports on a New York City couple, Sheila and her husband Simon, disguised themselves as members of the opposite sex and spent time walking the streets of New York. Immediately, Simon observed that "being a woman means being continually noticed and assessed" (p. 319). He also experienced anger and frustration towards comments that were sexual in nature. On the other hand, his wife Sheila "enjoyed the anonymity of not being stared at for a change" (p. 319). Sheila felt as if she had the ability to take up space in public spaces rather than experience fear for taking up space. This experiment demonstrates that role reversal does not change the way individuals experience unwanted effects and feelings as a result of catcalling.

Women's experiences of catcalling are also silenced as a result of their responses to catcallers. Some women indicate that they are grateful for catcalls because they enjoy the attention they are being afforded. Other women's responses to catcalling tend to be 
unconstructive as they attempt to ignore the situation as a result of annoyance or the desire to avoid rewarding the catcaller by responding (Chhun, 2011). It is also possible that women ignore the behavior as a result of experiencing a sense of embarrassment in regards to the degrading manner in which they are being treated. This leads to the internalization of feelings of humiliation as well as repressed anger.

Scholars contend that women's varied responses to catcallers result from socialization as women are taught not to respond. Tuerkheimer (1997) asserts that throughout women's lives, they have been socialized to keep silent rather than confront others (p. 192). Thus, women are not "socialized to react to abusive language by engaging in a physical fight" (Chhun, 2011, p. 290). Women are socialized to not respond, which contributes to their hesitation when attempting to confront catcallers. As a result, silence tends to come naturally to women as it is unlikely that women will confront catcallers in an aggressive manner. Confronting harassers would require women to "somehow leap out of our social context and become instantly "male" (Tuerkheimer, 1997 p. 192).

Although scholars have determined that women may lack the necessary socialization to engage in such confrontations, some women confront their catcallers. Rawlins (2012) conducted in-depth interviews with 18 undergraduate women attending college at a small liberal arts school in New York City. The interviews explored participants' study-abroad experiences in relation to public harassment and catcalling. Seven students traveled to France and four to Spain. Two students studied in "London, two in Mexico, and one each in Ireland, Italy, and Germany" (p. 482). When participants 
experienced instances of public harassment, more specifically catcalling, they refrained from responding in a way that would elicit forms of violence enacted by the perpetrator. Participants also expressed feeling frightened in situations "even though the behaviors they were confronted with what may have been only comments on their physical appearance or catcalls" (p. 489). These women then expressed response strategies that were utilized to minimize feeling fearful in public spaces. Rawlins identifies a "successful response strategy to catcalling" as one that solely involves verbal comments but also minimizes emotional distress for female victims (p. 489). Even so, most women ignored the comments except for one participant who had previously experienced sexual assault and angrily confronted male catcallers. Ignoring catcalls often leads to an intensification of the catcaller's actions as the man is more apt to respond with aggressive tactics such as violence or assault. Thus, women's refusal to respond can potentially result in hostility such as forcing women who ignore catcalls to undergo even more antagonism (Chhun, 2011). Conversely, women who do not respond to catcallers appear to condone this objectifying behavior, which encourages the behavior to persist.

\section{Feminist Standpoint Theory}

Feminist standpoint theory is one of many theories that stem from the broader category of standpoint theory. Formulations of this broader theory stipulate that standpoints arise when individuals are able to recognize the power relations in society that oppress particular groups. Feminist standpoint theory is identified as a critical theory as it aims to bring about awareness of marginalized groups while challenging social hierarchies and the consequences of their established dominance in society. This theory is 
rooted in Marxist ideology, specifically the notion that the working class's unique standpoints differ from that of the ruling class. The ruling class's "ideas are widely inculcated and presented as objective, advancing the view that social conditions are as they are, and cannot be otherwise" (Ratliff, 2006, p. 335). This establishes the ruling class's privilege within society. The dominant group often fails to recognize or understand the subordinate group's perspective. As a result, feminist standpoint theorists have suggested that the dominant group has less knowledge of the social world. (Wood, 2008, p. 397). Any feminist standpoint brings about some aspect of social totality which has "been previously suppressed with the dominant view" (Harding, 1986, p. 154).

Sociologist Dorothy Smith and political philosopher Nancy Hartsock were major contributors to the evolution of feminist standpoint theory and its involvement within the communication discipline. Hartsock's 1983 book titled Money, Sex, and Power changed the course of feminist theory by emphasizing the importance of women's lives as they "differ systematically and structurally from those of men" (p. 231). Because women's lives differ systematically from that of men's, women's standpoints are unique as they understand the world "from the perspective of the socially subjugated" (p. 149). As in Marxist thought, feminist standpoint theory asserts that the socio-political positions of women are marginalized by those who occupy the privileged positions in society. Women, like the working class, possess a distinct set of experiences and knowledge that allows them to see the world differently from the dominant, male-biased world.

The principle claim of standpoint theorists is that the positions occupied by women are starting points for investigating both the groups that are socially marginalized, 
as well as the groups that are socially privileged. The groups that are socially privileged hold positions that inherently oppress particular social groups. As a result, women's understandings of the social world shape their experiences, but they do not automatically signify a feminist standpoint. In order to "develop a feminist standpoint, individuals must engage in an intellectual struggle to recognize, analyze, and contest broad power relations that account for the subordinate status of girls and women" (Wood, 2008, p. 397). Learning a standpoint requires an individual to attain a level of intelligence that comes from political awareness and a person's lived experiences. The goal of feminist standpoint theory is to highlight the knowledge that arises from women's experiences and the activities normally assigned to females. It is valuable to understand the features of women's experiences because they are often disregarded. One is able to recognize women's experiences by acquiring and understanding women's interpretations of their lived experiences. By focusing on experiences, standpoint theorists are incorporating Marxist theory in that "the concrete activity in which we engage — shapes what we know and how to behave" (Wood, 2008, p. 397).

Feminist standpoint theory is based on two foundational issues: the notion of truth and the role of external forces in shaping standpoints. First, feminist standpoint theorists reject the notion of an unmediated truth, "arguing that knowledge is always mediated by a host of factors related to an individual's particular position" (Hawk, p. 536). That is, one's class, race, and gender construct one's conceptualization of reality. Even though feminist standpoint theorists renounce the possibility of an unmediated truth, "feminist epistemologies do not reject the notion of truth altogether" (p. 536). Certain social 
positions can produce particular views of reality as in a privileged or an oppressed perspective. These perspectives cause individuals to develop a particular view and understanding of the world around them.

Furthermore, feminist standpoint research is predicated on the notion that individuals' standpoints are shaped by external forces (Dougherty, 2001, p. 374). Knowledge stems from social ideologies, contexts, and men and women's engagement in particular activities. Men and women are allotted different opportunities within society, which leads to the co-creation of different role expectations. Such role expectations shape men and women's different understandings of social issues such as catcalling. In terms of catcalling, men are more likely to form standpoints around the role of the harasser, while women are more likely to form standpoints around the role of the victim (p. 374). Feminist epistemologies are thus "grounded in those shared characteristics of women as a social group" (Harding, 1986, p. 162). Even so, knowledge is by no means a "straightforward outcome of essential characteristics of group members (for instance, an XX chromosomal structure or having ovaries)" (Wood, 2008, p. 397). Standpoints express women's understandings of the social world while simultaneously exposing the social structures that men have created (Hartsock, 1983, p. 246). Therefore, women's knowledge and understanding of catcalling may stem from their gendered experiences as recipients of catcalling behaviors, while men's knowledge may stem from their enactment of catcalling behaviors. Even so, it is possible that women also catcall men in which these roles could potentially be reversed. Further research needs to be conducted to examine possible factors that might influence women's likelihood to catcall men. 


\section{Summary and Research Questions}

Wood (2008) acknowledges the importance of giving voice to women's experiences and the meaning that women attach to those experiences. Giving voice provides individuals with visibility and social standing in regards to "what had been invisible and thus had no social legitimacy" (p. 323). As these terms like sexual harassment and date rape were finally coined within the English language, theorists were able to raise awareness in regards to women's experiences and knowledge, which had been historically undervalued compared to men's perspectives (p. 328). This then validated women's experiences and provided insight into their gendered lives and their experiences of coping and resisting oppression (Foss \& Foss, 1994, p. 39). Based on the necessity of giving voice to women, we must gain a richer understanding of women's experiences of catcalling by applying Feminist Standpoint Theory to the current study. Specifically, it is important to give voice to women who have been affected by catcalling as well as the emotions that women experience and express after being catcalled. The current investigation will address five important research questions designed to achieve this goal.

First, as we have seen, researchers have utilized interviews to explore women's experiences of street harassment and women's experiences of street harassment when studying abroad. Gardner (1995) interviewed 293 women and found that women felt vulnerable and objectified as a result of experiencing street harassment. Unlike Gardner's study, the present study will utilize a theoretical framework to look at a specific form of harassment, catcalling. It is likely that women's experiences of catcalling differ from 
experiences of street harassment because harassers enact very different behaviors. This study will also differ from Rawlins' (2012) study, as experiences abroad are likely to be very different from experiences in the United States as street harassment is often normalized in other countries. The following research question was posed to address these research gaps and to identify how women define catcalling in their own words.

RQ1: How do women perceive or interpret their catcalling experiences?

Second, studies have yet to explore women's emotional responses to catcalling. Previous research emphasizes that stranger and street harassment elicit feelings of emotional distress (Bowman, 1993). Both forms of harassment evoke feelings of anger, fear, and disgust. McCarty, Iannone, and Kelly (2014) determined that behaviors perpetrated by a stranger elicit more negative emotions compared to a known harasser. Although these findings are noteworthy, the researchers only mentioned "negative emotions," while failing to recognize specific negative emotions that participants expressed, such as fear and anger. Also, it is possible that some targets of catcalling —or targets of some catcalling - may experience some positive emotions at the same time. In sum, researchers have been ambiguous when identifying women's emotional responses toward sexual, stranger, and street harassment. This lack of research signals a need for scholars to learn more about how women feel emotionally after being catcalled. The second research question was developed in order to address this lack of research.

RQ2: What emotions do women feel in response to catcalling?

Third, researchers have determined that women either actively or passively respond to harassers. Media outlets have demonstrated that women who actively respond 
to street harassers often experience violence as a result of their communicative responses (Feis, 2014; Lambertz, 2014). In terms of stranger harassment, Fairchild and Rudman (2008) determined that women who confronted harassers experienced less selfobjectification compared to those who passively responded and reported feeling sexually objectified. Women's communicative responses have been explored in regards to street and stranger harassment. Even so, studies have yet to examine women's communicative responses to catcallers, specifically. Therefore, the third research question is posed.

RQ3: How do women communicatively respond to catcallers?

Fourth, as previously demonstrated, contextual factors such as attractiveness and level of threat influence women's perceptions of stranger harassment. Fairchild (2010) found that an attractive perpetrator's actions were viewed as less threatening than an unattractive perpetrator. Time of day, location, and the age of perpetrators influenced participants' perceptions of perpetrators. Researchers have also examined situational factors that influence men's enactment of sexual, stranger, and street harassment. Group norms increase men's likelihood to harass victims as such norms lead to a sense of anonymity (Pryor \& Stoller, 1994; Wesselman \& Kelly, 2010). It is possible that other factors such as the attractiveness of the woman might influence the actions of the perpetrator. Current research has yet to examine contextual and situational factors that might affect women's perceptions of catcallers. The present study utilizes Feminist Standpoint Theory to explore these factors, as observed in following research question. RQ4: How do contextual or situational factors inform women's perceptions of catcalling? 
Finally, research studies have indicated that stranger harassment is not always viewed as an offensive means of insulting a woman. Fairchild (2010) indicates that women are often conditioned to view stranger harassment as complimentary. Women's experiences are trivialized as they are unable to communicate the harmful nature of stranger harassment. Researchers have also demonstrated that men believe catcalls are a means of complimenting a woman. It is likely that women's experiences of catcalling are trivialized as a result of men's belief that catcalling is complimentary, which is addressed in the fifth research question:

RQ5: How, if at all, do women think that their experiences have been trivialized?

\section{Conclusion}

The phenomenon of catcalling has received little scholarly attention except when discussed in relation to street harassment. This limits women's abilities to discuss their perspectives on catcalling as well as to describe specific instances in which they have been catcalled. In order to combat this lack of knowledge surrounding the topic of catcalling, research must examine women's standpoints on catcalling so as to illuminate this phenomenon within feminist research. This will assist women in "developing strategies to make sense of and make choices about the world in which they live" (Foss \& Foss, 1994, p. 42). It is also possible that naming the phenomenon will assist men in making sense of and making choices about whether or not to engage in catcalling behaviors. Overall, naming the phenomenon might make women's catcalling experiences visible within the legal realm, and women might be more apt to communicate their 
experiences with others. This will give voice to women while simultaneously shedding light on a topic that has been, and continues to be, trivialized in our society. 


\section{CHAPTER III}

\section{METHODS}

Women's experiences and perceptions of catcalling are under-researched. As a result of the high number of women who are targets of sexually suggestive comments every day, such experiences merit our attention. In the previous chapter, I introduced Feminist Standpoint Theory as a framework for the current study. The chapter also discussed literature pertaining to forms of harassment such as sexual, stranger, and street harassment, finally examining catcalling in order to identify gaps within current research. The purpose of this study is to reveal perceptions of catcalling from the standpoints of women, which are currently overlooked within communication research.

\section{Participants}

The 13 female participants I gathered for this study were college aged women from small or mid-sized Midwestern communities. Ten participants were Caucasian and the ethnicity of the other three participants is unknown as they participated in phone interviews. Since men are normally the sources of such harassment and women the victims, women were chosen for this study in order to provide an examination of their experiences as targets of harassment. Participants were required to have experienced some form of catcalling by one or more males. In terms of age, participants ranged from 19 to 26 as my study required that participants were at least 18 years of age. Although 
this study was not targeting a specific age, a range was implied by the requirements that participants must be women who have experienced catcalling. Since researchers have yet to examine how women's relationship status impacts their perceptions of catcalling, I found it necessary to ask participants about their current romantic relationship status. Of the 13 participants, 7 were single, 4 were in committed relationships, and 2 were engaged. I collected interview data until redundancy was achieved. Redundancy is conceptualized as the repetition of themes within data analysis until no new themes emerge (Lindlof \& Taylor, 2011). I achieved redundancy after the first 9 interviews, but I continued collecting interview data to give voice to women who had contacted me about participating in my study. These 4 interviews revealed no new themes, which confirmed that my saturation point had been reached.

In order to collect participants, I utilized a self-selection sample and a snowball sample. First, the university sent out an email invitation (APPENDIX A) via intercampus mail to a percentage of the student body. Students who indicated a willingness to participate in studies through the university were able to participate. The email included the general purpose of the study. Second, I used a snowball sampling technique. Participation was elicited through Facebook by posting a call to research. The call encouraged others to pass on the message or share the Facebook post. Provided the participants met the criteria, individuals who wished to volunteer were instructed to contact the researcher via email. I did not ask friends or acquaintances to participate in the current study in order to avoid coercion. 


\section{Data Collection}

The present study used a qualitative research design in hopes of gaining an understanding of women's lived experiences, their perceptions of catcalling, and their communicative responses towards perpetrators. Researchers utilize qualitative interviews to obtain "descriptions of events that are normally unavailable for observation" (Lindlof \& Taylor, 2011, p. 3). In doing so, qualitative researchers are able to establish rapport with participants thus making them more comfortable throughout the interview process. In the current study, qualitative interviewing proved useful because it provided an indepth understanding of women's catcalling experiences

In a previously mentioned study, Nielsen (2000) conducted in-depth interviews "to learn how the subjects experienced such interactions, not simply how they responded" (p. 1062). Because the researcher utilized this method in regards to a sensitive topic such as street harassment, I found it essential to use qualitative interviews to examine another sensitive topic, catcalling. By using interviews for the current study, I was able to construct as complete a picture as possible of women's standpoints on catcalling as well as their conceptualizations of catcalling behaviors as complimentary or harassing.

\section{Procedure}

I utilized in-depth interviews in order to explore participants' personal experiences. Each interview was conducted in a one-on-one context and lasted between 30 to 60 minutes, which gave participants a sufficient amount of time to discuss their experiences. Specifically, respondent interviews were utilized to elicit open-ended responses from participants. Respondent interviews, as indicated by Lindlof and Taylor 
(2011), "are conducted to find out how people express their views, how they construe their actions, and how they conceptualize their life world" (p. 179). Respondent interviews were particularly appropriate for the current study because participants disclosed their subjective standpoints on catcalling. Participants' narratives are also included as they were asked to identify their responses to catcallers, which were discussed in the form of narratives (see APPENDIX C). Three of the 13 interviews were conducted over the phone. In-person interviews were conducted in a location chosen by the participant to ensure she felt comfortable throughout the interview process. I recommended the interviews be conducted in a protected environment such as participants' homes, which helped participants from feeling preoccupied or stressed. I made participants aware that if they chose a public location for the interview, I could not guarantee that anyone who overheard the interview would maintain confidentiality.

\section{Interview Protocol}

Before the interview process began, participants read and signed an informed consent statement (See APPENDIX B). The informed consent provided information about the purpose of the study, confidentiality, and the underlying risks of participating. The consent form included my contact information for participants to utilize in terms of any questions, comments, or concerns regarding the study. Once participants were aware

of the risks associated with participating in this study, they participated in semi-structured interviews. Semi-structured interviews are relaxed and informal in nature as information discussed in the interviews emerges from a flow of conversation (Lindlof \& Taylor, 2011). As the interviews resembled a conversation, participants had ample time to 
respond to questions that were asked from an interview guide (See APPENDIX C). Supplemental questions were also employed at my discretion. Because the interviews were semi-structured, I had the freedom to discuss certain issues in a different order based on participants' responses. This allowed the interviews to flow, which made participants feel as if their responses were voluntary rather than forced.

Specifically, the questions that guided the interview are as follows. First, participants were asked introductory questions as well as demographic questions in order to make participants feel more comfortable. These questions also allowed for an understanding of the participants' education and relationship status. Next, I transitioned into participants' experiences with catcalling. These questions asked participants to recall a specific time in which they were the recipient of a comment from a male stranger in a public space. They were asked to describe if they responded, and, if so, how they responded, as well as how they felt during and after that experience. Participants also discussed whether or not they communicated their experience with others. Participants who communicated their experience were asked to discuss their motivation for doing so. After inquiring about their experiences, I asked participants to define a catcall. Once participants had described their own definitions of catcalling, I provided Chhun's (2011) definition of catcalling. Then, I inquired about participants' perceptions of catcalling. As catcalling is often perceived to be complimentary, offensive, or a combination of both it was necessary to gather participants' interpretations of catcalling. Participants were asked to describe a hypothetical situation in which they would consider a catcall to be complimentary or rude. Questions also addressed potential circumstances that might 
make a catcall more or less offensive. I did not refer to catcalling as harassment until the last question because in doing so, participants might have felt more negatively towards catcalling, which could have swayed responses.

\section{Data Analysis}

Each respondent interview was audio-recorded and transcribed verbatim. All audio files were kept on a password-locked file on my personal computer. Each interview was transcribed word for word in order to ensure the accuracy of participants' responses. In order to protect participants' identities, I assigned pseudonyms to each participant to ensure confidentiality. After transcribing the interviews, I conducted a thematic analysis to understand participants' experiences as well as to describe the data in rich detail. Thematic analysis is defined as "a method for identifying, analyzing, and reporting patterns (themes) within data" (Braun \& Clarke, 2006, p. 79). Specifically, I attempted to discover repetitive themes from participants' responses. A theme "captures something important about the data in relation to the research question, and represents some level of patterned response or meaning within the data set" (p. 82). It is important to note that themes emerged from participants' experiences of the subject matter rather than the researcher's understanding of the phenomenon. After the first 5 interviews, I coded for themes, and I continued to code as I collected interview data from the remaining interviews.

For this study, I applied the methods of unitizing and categorizing. Unitizing involves breaking data into discrete sequences. The researcher essentially separates information from interview transcripts into "single pieces of information that stand by 
themselves, that is, that are interpretable in the absence of any additional information" (Lincoln \& Guba, 1985, p. 203). Particularly, I sought units that pertained to the research questions. I then applied the method of categorizing in which the unitized data was organized into categories that provided "descriptions or inferential information about the context or setting form which the units were derived" (p. 203). I utilized categorization by regrouping the units along similar themes that were relevant to the research questions, also known as domain analysis. Domain analysis can be defined as a description of a category or domain by means of semantic relationship (Baxter, 1991). That is, the researcher is looking to define "the set of Xs that are kinds of Y, parts of Y, causes of Y, effects of Y, reasons for doing Y, ways to do Y, and so forth" (Spradley, as cited in Baxter p. 245). By utilizing domain analysis, I was able to develop a richer understanding of women's catcalling experiences as well as their perceptions of the harm. In order to achieve external validity, I provided a thick description of my participants' responses to ensure for trustworthiness. Lincoln and Guba (1985) describe a thick description as a way in which a phenomenon is described in sufficient detail, which allows for conclusions to be drawn that are transferable to other situations and people. By providing detailed accounts of participants' responses, one is able to begin interpreting participants' motivations and intentions that characterize their experiences. Also, by having all of the first interviews coded and transcribed, but continuing to collect data after reaching redundancy, I utilized a type of "referential adequacy," in that some data were "set aside" and only coded after all categorization schemes were completed (Lincoln \& Guba).As a feminist researcher, I attempted to shed light on women's experiences while expanding 
my own knowledge of the subject matter. More importantly, the goal of this study was to bring about awareness in regards to the often threatening nature of catcalling. Specifically, for research question one, I looked for units pertaining to women's understanding of catcalling as complimentary or offensive. For research question two, I looked for units that indicated certain emotions or emotional responses to catcalling. For research question three, I looked at how women communicate with catcallers using verbal or nonverbal communication. For research question four, I examined units pertaining to women's perceptions of the contextual factors that give meaning to specific instances of catcalling. I also looked at how women's perceptions are affected in terms of the time of day in which the catcall occurred, the location that the incident took place, and the attractiveness of the perpetrator. Finally, for research question five, I looked for participants' communication of their experiences with others as well as participants' description of their experiences as trivial.

\section{Conclusion}

In conclusion, semi-structured interviews allowed me to develop possible themes for women's perceptions of catcalling as well as their conceptualization of catcalling behaviors as complimentary or offensive. Essentially, this method provided an in-depth understanding of participants' experiences by allowing me to give voice to their stories. By conducting a thematic analysis, I organized emerging themes throughout the interview process that pertained to women's standpoints on catcalling. The following chapter demonstrates the findings that coincide with the research questions previously stated. 


\section{CHAPTER IV}

\section{FINDINGS}

In the preceding chapter, I discussed my methodology by highlighting information regarding my semi-structured, in-depth interviews. The 13 interviews I collected lasted between 30-60 minutes. Once I transcribed all 13 interviews verbatim, I coded participants' responses into representative themes. In this chapter, I will discuss the emergent themes as they pertain to my five research questions. First, I will discuss how women perceive and evaluate their catcalling experiences.

\section{Women's Perceptions of Catcalling}

The goal of research question one was to understand how women perceive and make sense of catcalling. Women defined catcalling as a man yelling sexual or derogatory comments towards a woman. Although many of my participants emphasized that men were the perpetrators, four participants specified that anyone could engage in these behaviors. Catcalling was also described as primarily verbal and audible, while 4 participants identified that it might also involve nonverbal behaviors such as staring. Many of the women mentioned that a catcaller's use of particular words or phrases influenced their perceptions of catcalling. Certain comments were viewed more negatively (e.g., "hey sexy"), while others were viewed positively (e.g., "you look good"). As a result, women did not always describe catcalling as a primarily positive or 
negative experience. Some women disclosed that their perceptions not only stemmed from previous experiences with catcalling, but other influential factors such as the values that family members had instilled.

\section{View of Catcalling}

Many of my participants defined catcalling similarly. Riley explained her definition of catcalling as, "Any like male stranger who's yelling something." In the same way, Gina described catcalling as a man shouting something, but she made sure to emphasize that men usually yell "something at you that's inappropriate or sexual." Aside from some women's indication that anyone could engage in these behaviors and the behaviors could be nonverbal, the views were pretty consistent. In terms of women's evaluations of catcalling experiences, several participants clearly identified catcalling as an overall negative experience. On the other hand, some women described catcalling as a partially negative experience, but if a catcaller used certain words, women evaluated the catcall more positively. This section will first highlight women's view of catcalling as a negative experience. It will then move to the comments women identified as positive.

Comments about my body. The larger number of participants mentioned that comments about a woman's body were rude and offensive. Some women emphasized that these comments went "too far" because they identified particular body parts. Lindsey described a particular experience with a man commenting on her body:

There was a group of African-American males. As I was walking out the door, they said, "Ooo look at the ass on that one." And I was just like, "Okay, one I have a name, two, you said that to where everyone can hear, including me and my 
friends and, three, that's not a really appropriate way to show that you're interested in me or attracted or whatever he was trying to do." I don't know if he thought that was going to work? Like I was going to turn around like, "Oh yes here's my number. You just referred to me as 'the ass on that one."

Later on, Lindsey related back to this experience and emphasized that the men were "being so rude," and she would rather have them look at her body rather than verbalize their thoughts. Similarly, Katie mentioned, "I don't want a stranger talking about my body quite frankly." She then described the catcall "look at that ass" as a comment she found to be derogatory and rude.

Some women not only identified comments about body parts as rude or offensive, but also comments regarding what a woman should wear. Diana illustrates this:

So you know, like I said, the physical, like your boobs or your butt or your body or what you're wearing. You know I hear a lot of people like a lot of boys say, "Wear something tight" and stuff like that. I feel like that's very rude and offensive.

"It was a stranger." Because catcalling can involve a stranger making comments towards a victim, women often viewed it differently compared to a known catcaller. Twelve of the 13 participants emphasized that comments from a stranger are much worse. This theme echoes McCarty, Iannone, and Kelly's (2014) study as they found that known harassers are granted more leeway, and the harassing behavior is viewed as less threatening. On the other hand, the behavior of stranger harassers is regarded as more severe, resulting in more negative reactions and outcomes for victims. Many women 
identified lack of personal acquaintance as the primary factor in which the catcalling behaviors were inappropriate and negative. Gina, for example, compared a close friend yelling at her to a stranger:

The audacity of someone to yell something like that, unless like I said, unless you're like a close friend and it's a joke and you know the intention of it is to make a joke. But if you're a stranger to that person, even if you're an attractive person, I still think that's very unattractive to do that.

Here, Gina is more accepting of a friend's behavior because his intentions are seen as harmless. If a stranger performed similar actions, his actions would be viewed more negatively or even "unattractive."

Similarly, Maddie described her perceptions of a stranger's behavior compared to that of a friend's in terms of relational closeness:

It's a stranger with a new person, so there's a level of - - d don't know-I like to think of it as like rings. Like kind of strangers on the outside of the ring and then as you move in closer to the center it's like your friends and family around that ring. So I think that the degree of separation or the degree of relationship and delivery are the two things...because I think that if my friend, was like a good guy friend, was like, "Oh you like have really nice heels." "I can see your boobs" or you know just something very...I feel like I wouldn't take it as like catcalling or offensive, but if it was a stranger who said that exact same thing. I think that just gives a whole different connotation to it. 
Objectifying. As stated in the review of literature, Fredrickson and Roberts' (1997) objectification theory asserts that when women are objectified, they are essentially being treated as a body, as if their bodies exist for others. Several women expressed that catcalling made them feel like objects or "pieces of meat," which coincides with the main tenet of objectification theory. Blaire asserted that catcalling is "negative all around" because a catcaller's comment about a woman's appearance puts her on display as an object upon which to comment:

I feel like there's this underlying-like, "I'm entitled to you as a woman"-like "you are...not just there but I'm going to comment about your physical appearance and ogle, and you're just on display like a, you know, piece of art and so that I can make those comments and it's justified because it's laced with this niceness."

Allie discussed how women who enjoy catcalling misconceive it as a positive manner for males to give women attention. Women who view catcalling positively are encouraging men to treat them as objects:

Because you are getting attention for that, and if that's what you want, you're getting it, but you're also reinforcing the fact that you feel like you're a sex object. I think it's definitely misconceived to be a positive thing. I don't think it's a combination. I think an informed perspective would be able to distinguish one from the other.

Paige also viewed catcalling as a negative experience, but she struggled with clearly identifying it as an overall negative experience. She was one of a few participants 
who felt like some verbal remarks could be viewed positively. As in Fredrickson and Roberts' (1997) objectification theory, there are also positive effects of sexual objectification. Physical beauty often translates into power as more attractive women are treated more favorably socially and economically. Catcalling led some women to feel as if their level of attractiveness had been positively reaffirmed. Paige emphasized that she viewed the comments positively for a few seconds until she felt objectified:

I think I've experienced that before when I'm older especially then I'm like, “Oh okay yup I'm attractive." I'm proud of that in a weird way because society has made me think, "Okay well you should be desirable as a woman." But then it's a weird automatic flip into that anxiety of like...'I'm glad you think I am but now I'm afraid of what you're going to do because you think of me like that" or "you're obviously yelling at me as somewhat of an object."

When discussing the verbal remarks she viewed as rude and offensive, Lindsey identified that comments about her appearance such as her "ass" and "lips" made her feel like she was not an individual. Lindsey also discussed how comments such as "hey mama" make her feel like an object:

I guess it itemizes me. There's that one, "hey mama." I'm my own person and you say...like I know you don't know my name. But you still don't have to refer to me as like a thing. As if I don't matter, an object.

Harassment. A theme that arose in regards to this research question was that several women identified catcalling as a form of harassment. Factors such as the severity of a catcaller's comments, the minimization of women's perceptions of catcalling 
behaviors as offensive, and persistency influenced participants to consider catcalling as a form of harassment. Even so, women did not identify catcalling as harassment until they were asked to disclose their perceptions of catcalling as harassment. After doing so, participants discussed that catcalling can be seen as harassment, especially if it makes them felt uncomfortable or threatened. Katie described that for her, classifying catcalling as harassment is dependent on the catcaller's behaviors. If she felt offended by the behaviors, she would consider that to be harassment:

If they're staring at my ass, I would feel a little harassed or violated for that matter. If they said something about my body, looking at those particular body parts, yes. But just a whistle I wouldn't really say so. It would just depend on the type of remark. Like I said if it's something that offended me, absolutely.

Although Katie would consider certain catcalling behaviors as harassment, she did not classify catcalling itself as harassment. She made sure to emphasize that, for her, not all catcalling behaviors are negative; some can be positive, which coincides with her take on catcalling as a form of harassment.

Lindsey, along with several other participants, communicated disapproval towards a catcaller's failure to recognize the effects of his actions. She identified a hypothetical scenario in which she told the catcaller, "That makes me feel uncomfortable," and he responded with, "Oh come on chill out." For Lindsey, this was harassment because it minimalized her feelings. She went on to say, "You don't get to decide if you hurt someone's feelings or bother them." 
Many of my participants felt that persistence was the main factor that led them to identify catcalling as harassment. In hypothetical scenarios, participants described persistence as a catcaller "relentlessly" making comments towards them and then continuing to follow behind them regardless of whether the participant communicated her disapproval of the behavior. Diana asserted that catcalling "could be harassment if they don't stop after just saying one thing if they keep on going on. I think that's harassment." Ella also made the distinction between viewing persistence as harassment but not a single comment:

I think it's hard to say....when there are so many different definitions of catcalling because in certain cases yea it could be harassment. You're harassing somebody on the street if you're not letting them go like the persistency. That feels like harassment but a single comment...I don't know if I really see it like that. Ella felt as if catcallers could definitely cross the line if they were persistent, but she was clear to state that "it's hard to make a line because what might feel like harassment to somebody else might not to me."

Different ways to give a compliment. Another major theme in relation to research question one involved participants disclosing that catcalling was not a compliment. Eleven of the 13 participants verbalized that it was not a compliment. Riley made a clear distinction between catcalling and what she views as complimentary:

If someone's just like giving me a compliment in, like, a non-threatening way and they don't want anything else from it, they don't expect anything from it, then I 
take it as a compliment. When I think catcalling, I always think of it in a negative light, not in a complimentary way.

Blaire also indicated that she would not view catcalling as complimentary. Even if she viewed the behavior as harmless and the person had "a hundred percent positive intentions," the comments are unwarranted because they are essentially interrupting women "trying to safely navigate through a public space." For Blaire, when men catcall women, "there's always a hidden agenda masked with a compliment."

Several women also emphasized that men misconceived their comments as compliments. Paige illustrates this:

I think that they might think of it as a compliment but that's why they kind of have it...if they say, "I'm not being offensive, I'm calling her attractive." It's like okay, but the way you call her attractive — there are a lot more options that you can do-if you genuinely think a woman is attractive.

Paige used verbal comments such as "hey beautiful" to demonstrate comments that men might view as compliments. She went on to say that regardless of the catcaller's perception of catcalling as complimentary, their comments are "inappropriate and offensive.”

After indicating that catcalling was not complimentary even though men might view it as such, many participants described a strategy men could use to compliment women. These included approaching a woman in a respectful manner to start a conversation rather than yelling at her from across the street. Mary referred to a specific experience with catcalling in which she had been walking on a college campus in the 
morning. She was walking to class wearing a "button down shirt and a skirt." It was windy so Mary dropped something on the floor, went to pick it up, and three men started making comments such as “you better not bend over too much otherwise you're just asking for it.” In response to her experience, Mary indicated:

If you want to talk to me, and if you think that I'm pretty, why don't you tell me that? Or if those guys that day wanted to talk to me, couldn't they say, "Hey you look nice today!" You know what I mean? You can get my attention in different ways that is respectful to who I am as a person not because I'm wearing a short skirt.

Positivity of "you look good." Even though women often described catcalling as a negative experience, some participants mentioned that there is a small window in which catcalling can be positive. Comments that refrain from identifying body parts such as "you look good," "you look nice today," and "wow" were regarded as positive catcalls. Lindsey demonstrated when she might view catcalling positively:

If I put in a lot of time getting ready, one of those days like top to bottom, your hair, your makeup, shaved legs all that good stuff, find your favorite dress and put it on. And I would be walking down the street and someone was like "Wow" or maybe some type of an expression like. I would probably be like, "Okay, I'm doing something right now that you noticed me."

Similarly, Paige described comments that are not "explicitly vulgar" such as "hey you look good day" as positive comments, in which a small part of her thinks, "Well I'm glad I'm attractive." Some women disclosed that positive catcalls result in a "boost of 
confidence" or that they enjoy the attention. Leslie described that "at times it kind of feels good to be noticed" because a part of her likes the attention.

Several participants emphasized that they did not personally view catcalling positively, but other women might view comments such as "you look good" as reassurance that they are attractive, which might boost a woman's self-esteem. Ella highlights why a woman might appreciate a catcall:

Maybe girls that do enjoy that aren't getting the type of appreciation from people that actually respect them. You know, so maybe they aren't getting those types of comments in a more polite way. So when they hear it, too, you know, when somebody like me would see that that's rude, but they don't see it that way because they're not used to just being respected.

\section{Making Connections to the Past}

Another domain in accordance with research question one has much to do with how women evaluate their catcalling experiences. Many of the women viewed catcalling in a particular way as a result of past experiences and how they were raised.

Past experiences. A theme that arose in regards to this particular research question was that women felt like their past experiences influenced their perceptions of catcalling. Upon reflecting on their experiences, women who had enjoyed catcalling at a younger age made sure to communicate that their current views were different. These women now view catcalling as an insult. Allie described how she used to view catcalling:

In high school, we would love that. We would see how many we could get. Like, we wouldn't do anything, but we would just count them all the time. It's 
unfortunate but we grow up thinking that it was like a compliment

Allie went on to say that how she viewed catcalling while growing up affected her current view of catcalling. Now, Allie feels "too old for that" and perceives catcalling as offensive.

Riley expressed encountering others who disagreed with her views of catcalling and how that influenced her perceptions of catcalling. She describes:

Me and my sisters have had conversations about it with my mom, I remember her telling me before like, "Oh sometimes people just need to learn how to take a compliment" and I remember being mad at her for that. I was like, "No it's not, like, that's not a compliment." Even if they could be saying something nice, but just the way they're saying it, that's not a compliment. So I think just my own experiences more than anything.

Mary disclosed that she had previously been in an abusive romantic relationship. When men catcall, Mary experiences flashbacks to the mistreatment, which impacts her current and future views of catcalling. She states, "What I went through in the past, and I never want to feel that again, like you know, be disrespected in that way, even if it wasn't to the extent of before."

How I was raised. Related to previous experiences, several participants mentioned how their parents, specifically their mothers, raised them to understand that men respect women, influenced their perceptions of catcalling. Women emphasized that men should respect women, "treat them like human beings," and not objectify them. Siblings also played a role in terms of participants observing their siblings' experiences 
and learning from them. Mary discussed how her mom impacted her perceptions:

A lot of it has to do with how I was raised, too, because my mom was born in Ireland, so she came over here when she was 16 and she had her own experiences with men and stuff because it's a different culture over there. So I think the way that I was raised from her kind of affected me, too. You know, I was raised, “Don't let anybody ever disrespect you." But I think for me it was so enforced because, you know, I was raised to stand up for myself at a very young age. Similarly, Katie described that she was "raised by very strong willed women" in her family and was surrounded by them when she was growing up. As a result, she was taught to stand up for herself and that "a gentleman looks you in the eyes and shakes your hand." Katie then described that how she was raised influenced her opinion in terms of being offended by men's comments about parts of her body.

Halle mentioned that her mom taught her brother that catcalling women was inappropriate and told him, "You don't objectify them, you don't treat them like that." Halle learned from her brother's actions in terms of how men were supposed to treat her, which influenced her perceptions of catcalling.

\section{Affective States}

The findings regarding research question one emphasize that women often evaluated catcalling negatively. This indicates the importance of examining women's emotional responses as they might express emotions that coincide with their evaluations of catcalling behaviors. Research question two regarded participants' descriptions of the emotions they experienced after being catcalled. Three categories of emotion or affective 
state emerged from the interviews. First, several participants identified the emotions of feeling "angry" and "annoyed," which are other-directed negative affect and, when expressed, function to correct the catcaller's behavior (Metts \& Planalp, 2011). Second, some participants described feeling "embarrassed," a self-directed negative emotion. Embarrassment is typically experienced when an individual violates social norms of appropriateness and, as a result, loses face during an interaction (Metts \& Cupach, 2008). In the episodes described by these participants, even though the victim did not violate norms, she experiences feelings of embarrassment vicariously because the catcalls bring unwanted public attention to her and compromises her ability to maintain the image of a respectful and poised woman. Finally, many of my participants identified the general affective states of feeling "uncomfortable" or "nervous." These affective states are related to fear as they are motivated by a desire to withdraw from the situation, especially if a victim is uncertain about a catcaller's intentions or potential actions.

\section{Anger}

Several participants mentioned feeling angry after experiencing a catcall. Anger is often expressed when a person's goals are thwarted in an unjust or unfair manner. Women would experience this affective state if they received a catcall when it was undesired and constrained their freedom of movement in a presumably open space which they expected to control, especially if the catcaller was persistent. Gina discussed situations in which catcallers would continue to pursue her. As a result, the catcallers' actions made her angry:

I was mad because, you know, usually it's like you can say one thing when you 
walk by, but these guys kept yelling things when we were halfway down the street. So, that kind of like made me angry, and then, I was kind of taken aback, too. My friends were like, "You shouldn't have said anything, blah blah blah"..."they could have, whatever, they could have come after us."

Gina also discussed how ignoring a catcall or giving a catcaller a dirty look prompted them to respond with, "Oh what's wrong" or, "Smile." In those instances, Gina expressed, "That makes me even angrier." For Gina, anger ensues when catcallers continue to make comments towards her to get a response, in effect, attempting to exert control over her actions.

Blaire discussed how her goal of walking down the street or to a friend's house is often thwarted because she is unable to do so without receiving catcalls. In response, Blaire feels angry:

That's when like the anger takes place. And that's what I get pissed off because like I'm just trying to walk down the street. "Can you just let me go from my car to my friend's house without you making a comment?"

Halle mentioned feeling angry, but as a result of men's behaviors and the fact that she does not consider catcalling to be complimentary:

I get pretty angry, like I'm not a piece of meat. And that's definitely how I feel when they whistle at me or say stuff. I think that if you want to compliment a woman there are much better ways to do it than whistling or yelling "hot damn" or things like that.

Annoyed. While some participants felt angry after being catcalled, other 
participants described the somewhat less intense feeling of being annoyed. Halle illustrates this:

It just annoys me because it's something that I have dealt with since like around puberty. So it's just annoying to get stared at or to get yelled at. You know, what if I did that to them. It's annoying. Like I can't get offended and say something to you because you said something rude and sexualized to me because that makes me a bitch or like a snot or ungrateful or whatever. It's very frustrating.

Halle discussed various experiences with catcalling. Whether she encountered a catcall on the street while walking to work or walking by a construction site, she said, "It's still annoying." She then stated, "I should be able to get from point A to Point B, walking, riding a bike, without getting catcalled.”

Diana described feeling annoyed when she would move to the other side of the street to avoid catcallers. She uttered:

Now that I think about it, it's kind of annoying because I don't like having ...I don't like anyone making me change anything that I'm doing. So that's kind of annoying that an act like that can alter what I want to do but yeah I don't know that kind of makes me like weak kind of.

\section{Catcaller Induced Face Loss}

Catcalling violates social norms, which can cause victims to feel embarrassed in response to the violation. Because catcalling violates social norms of politeness and face support in public spaces, victims experience face loss vicariously. Metts and Cupach (2008) indicate that embarrassment often occurs when people observe others' violations 
or inappropriate behaviors, and consequently, feel embarrassed for that person. Victims are not the source of their face loss, as is typical in most embarrassing situations, but they do experience the threat to public image caused by the catcaller's comments.

Embarrassment. Some participants expressed feeling embarrassed when other people were around to witness their catcalling experiences. Leslie disclosed an experience she had at a sports bar. After a work event, Leslie and her work colleagues went "out to a local sports grill and bar together." When they walked into the bar, she "got catcalled by a couple of guys in the bar" who yelled, "Hey, come over to our table." Leslie then described how that experience made her feel:

I kind of felt embarrassed, just because, you know, maybe if it was with a group of friends, I would have felt a little different. But since it was with work colleagues, it drew attention to myself in front of my work colleagues in a different way where I don't want that attention to be drawn to.

Here, Leslie felt embarrassed because she was with other people, specifically her work colleagues. If she had been with her friends, Leslie mentioned "I probably would have just brushed it off."

Gina described a similar situation in which she experienced a catcall while walking to work. She said, "I was embarrassed because there were professionals all around me and I was being yelled at with these inappropriate things." Gina also mentioned that she did not respond to the catcaller because a lot of professionals were around and she "didn't want to you know cause a scene or you know make more of a scene."

Lindsey also described feeling embarrassed with a lot of people around: 
Yeah, if there are a lot of people, then it's more embarrassing because you have so much attention right on you and the spotlight is on you. Yeah, that would - that would make a difference because there are more people. And I would not like that at all.

\section{Fear: The Desire to Minimize Harm}

Catcalling can often cause victims to feel "uncomfortable or "nervous." These affective states stem from the desire to minimize the harms that catcallers might induce, such as feeling objectified or degraded. Very often, the core of these affective states is not simply the discomfort of uncertainty and unpredictability, but the awareness of possible harm which induces varying levels of fear.

Uncomfortable. The majority of my participants indicated feeling uncomfortable when experiencing a catcall. For Lindsey, a catcaller's persistent comments made her feel extremely uncomfortable:

This guy wouldn't leave me alone. So it wasn't even, he had first like expressed verbal interest and said these things to me, and after I had told him like pretty politely like, no sir. I don't really appreciate this, he kept coming back and just kept trying. That's even ruder because I've already told you that I really like you to stop and then you keep coming at me and that's not okay. That makes me very uncomfortable.

Diana explained that catcalls make her uncomfortable because her brothers are very respectful, and she is not used to men engaging in catcalling behaviors. She identified catcalls as disrespectful as they make her feel uncomfortable: 
I would probably just feel uncomfortable. I mean I have like three brothers who are very just respectful, and I want respect for myself. So if I saw someone like talk to me like that or said something out of hand in front of me or just disrespectful, it makes me feel uncomfortable because I'm not really used to that. Maddie expressed that she felt more uncomfortable when experiencing catcalls in South America compared to the United States because catcallers would often pursue victims. She studied abroad in Peru and encountered catcalls on a daily basis: In Peru, they're actually going to be pretty descriptive of what they're saying in Spanish. They're going to be whistling more, they're going to be saying, "I want you." It's definitely more uncomfortable than I would say in the States because in South America there is definitely more deliberate like, "I'm going to try to touch you or I'm going to try to reach out to you," so definitely more uncomfortable in South America. I think that they have the confidence to actually pursue the catcall. They wouldn't just catcall and then just kind of walk away. It's a wild card of whether they're going to kind of follow. I'm more comfortable with the surroundings of the States than in South America.

Nervous. Some respondents described feeling nervous because they were unsure if the catcaller would act upon their comments or if their behaviors would escalate. After discussing feelings of nervousness, women often expressed being afraid or fearful as catcalling behaviors could become threating. For example, Paige disclosed an experience being catcalled when she was sixteen years old:

I had a dance studio that was probably a block away from a bar and when I was 
walking to my car from my dance lesson, I remember being yelled at or whistled at from the bar location. And it was dark at the time. It was probably like seven or eight. It wasn't anything extreme like ten/ten thirty when the bar would be very popular. But I remember getting yelled at, and, especially at such a young age, I was very nervous about. I was automatically very nervous about those males...there were multiple males... acting upon their comments. It made me very nervous, and I ended up rushing to my car and making sure it was locked because I was afraid.

For some respondents, the presence of other people brought on feelings of nervousness. Allie was catcalled while crossing the street with multiple cars and various people around. She expressed:

So, like, everybody heard it, and it was gross, and it was humiliating. I felt overwhelmed. I felt my cheeks get red and my throat tighten up. Because I was like, I don't know what to do, and I'm on the spot. So, I felt a little nervous. I felt like I was on a stage here for people in their cars.

\section{Communicative Responses}

Because women expressed primarily negative emotions and affective states in response to catcalling, it is important to explore how women communicatively respond to catcallers as their emotional responses might prompt particular communicative responses. Research question three pertained to women's communicative responses to catcallers. Particular factors influenced responses in terms of feeling more or less safe in situations. These factors also influenced the strategies that were used to respond to catcallers such as 
ignoring a catcall, avoiding the catcaller altogether, or verbally responding. Many of the women ignored catcallers, and very few verbally responded to the catcalls. After discussing their experiences with me through the course of the interviews, women expressed a strong desire to verbally respond and highlighted the importance of responding to catcallers in hopes of deterring future behavior.

\section{Strategies}

Women employed various strategies when responding to a catcall. Often, rather than verbal responses, these strategies involved ignoring or avoiding the catcaller. Women also expressed thinking back to their experiences, reevaluating them, and wishing they had verbally responded.

Verbal response. Several of my participants indicated that they responded to catcalls with verbal remarks such as "I don't like that" or "Yeah thanks." Halle disclosed a catcalling experience involving a group of construction workers building a hotel. They would yell things at Halle as she passed by the site "two times a day, seven days a week" on her way to work. Halle mentioned that she would respond by "flipping them off" or "telling them to fuck off." At one point she expressed feeling annoyed and frustrated, which prompted her to yell, "Do you talk about your daughters like this? Would you appreciate it if a guy was doing this to your wife?"

Leslie described verbal remarks she might make in response to catcalls:

I might just tell them, you know, "You can come out and tell me in a nice way that you think I'm pretty, but I would appreciate it if you did it in a different manner. Just the way you told me right now, you just wanted to come out and tell 
me I'm pretty... that's fine. Just make it not so offensive and not causing as much of a scene."

Ignoring the behavior. Another strategy many of the participants discussed was ignoring catcalls. This strategy was often used to communicate to the catcaller, "You aren't worth my time" or "I'm not interested." Women also described using this strategy to prevent the behavior from continuing because responding could result in a more threatening situation. Halle said, “I don’t yell stuff every time because that just attracts more negative attention. Then, they'll remember that that's the one that flipped you off." She went on to say that engaging with catcallers encourages them to continue the behavior. Halle said, "If you show that you're annoyed or that you're bothered by what they're doing, they'll just do it more."

Diana expressed her concern for responding to catcalls:

I mean I wouldn't want to react just because I know like, I wouldn't want to deal with that or you never know how the other person is going to react, do, or say. I honestly don't think I can fight a man, stuff like that. I just think they don't really deserve my time so I'm not going to give it to them.

Blaire used the strategy of ignoring catcalls as a result of her past experiences with responding. Instead of verbally responding, she used her silence to communicate that she had no interest in the catcaller or his comments. She uttered:

Yeah I just know I mean I have had a past of responding to those-not even just that, but just men in general when they're being kind of crude, and it doesn't really get you anywhere if you're alone especially. I've learned to kind of just use 
silence as a way to say, "I am not interested in you or what you're saying here or any of that." Yeah I just feel like especially in a situation where you're alone it could add to the danger of it.

This theme coincides with Rawlins' (2012) findings. Based on responses to qualitative interviews, participants refrained from responding to catcalls in hopes of deterring a catcaller's potentially negative or violent behavior. Just as Rawlins's participants, several of my participants expressed ignoring catcalls in hopes of preventing the situation from escalating.

Avoidance. Rather than ignoring catcalling behaviors, some participants felt the need to avoid catcallers all together. This often took the form of walking a different way, crossing the street, avoiding eye contact, or walking with another person in hopes that a catcaller would refrain from engaging in catcalling behaviors. After women discussed the strategies they would use to avoid catcallers, some expressed that avoidance was preferred but not an appropriate response. In particular, Leslie discussed that "avoidance isn't right" because "you basically have to go out of your way sometimes to not have that or put yourself in that situation." She then explained that avoiding catcalls is "tiring" and "frustrating" as it delays a woman's time when trying to walk somewhere.

Ella described a hypothetical situation because she was unable to recall a specific instance in which she avoided being the recipient of a catcall:

Sometimes I'm like, “Alright I'm just going to be confident. I know I'm wearing this really tight skirt right now or whatever it is. But I'm like I don't want to have to change what I do just to let them get on with their day. I don't want to change 
something I'm doing." That doesn't seem fair. I'm wearing something that people will look at me and want to make comments about, so I should do something differently. I should, I should avoid them.

Ella struggled with wanting to wear certain clothing, which meant receiving stares and comments. Instead of changing her clothes, she would take necessary precautions to avoid comments.

As previously discussed, Halle would make verbal comments towards the construction workers who were working at a construction site she passed on a daily basis. Halle expressed that some days she would completely avoid the street the site was on by walking down different streets:

Yeah, like sometimes I'm like, I don't want to deal with it today. I don't want to deal with what could happen. I just won't walk down the easiest way for me to get to work or wherever I'm going. Sometimes if I have a hood I'll put it up so then they can’t see my head or my face.

For Halle, some days it was easier to avoid the construction site all together rather than knowingly walk by the site and receive comments.

Blaire discussed crossing the street as a mechanism she would use to avoid catcalls:

If I see a large group like ahead of me and they're kind of being loud and obnoxious, I might try to like go across the street and just not really even put myself in that situation, but more times than not I'm not expecting it or it's unavoidable. 


\section{Factors that Influence Responses to Catcalling}

There were various factors that influenced women's decisions to utilize particular response strategies. Three major factors shared by my participants include, compliments turn offensive, deter future behavior, and safety in numbers.

Compliments turn offensive. Several of my participants indicated that catcallers' comments often moved from what a catcaller deemed "complimentary," regarding clothing or telling a woman to smile, to comments such as "slut" or "whore." More offensive comments occurred after women made comments back or ignored the catcalls. Halle expressed that responding to catcalls resulted in men calling her a "bitch." She described these experiences as a "lose-lose" because she would receive such comments regardless of whether she responded or ignored the behavior.

Ella referred to a hypothetical situation in which she ignored a catcall and the comments escalated:

Yeah it can turn into like an insult, too, if you walk away and they're like, "Oh you're too good for me?" Then that gets kind of offensive because it's like, "No. They're making you feel like you're the person in the wrong." So yeah that type of thing is offensive.

Riley discussed how her experiences with catcalling often escalated:

Because sometimes they'll even start out sounding like compliments, and then if you don't respond right way, or you just don't respond, it turns into stuff like calling people a bitch and asking if they swallow and stuff like that which is obviously not received as a compliment. So usually it escalates very quickly if 
you don't respond, then if you do respond, it just gets creepy.

After Riley discussed her experiences with situations that tend to escalate, she expressed, "There's really no right way to approach it, which is why you just keep quiet and then just keep walking."

Allie described a similar experience she encountered while walking across the street near a college campus:

There was a car of guys, and I think there was like four, maybe three guys, in the front and in the back. Their music was really loud, but one of them said something really degrading. I don't really remember what it was, honestly, but I remember that I gave them the finger. So I probably shouldn't have done that. And then, that spurred on, "F you, Bitch", "I'll fucking kill you", and "You're ugly", so that was over the summer. I was actually pretty surprised.

For Allie, the comments men perceive as "compliments," escalated to remarks that she indicated as "threatening" and "offensive." As a result, Allie felt "pissed off," which caused her to respond by flipping them off.

Safety in numbers. Another factor that some participants mentioned as influential when deciding to respond to a catcaller was that of being with a group of friends. Being with friends was described as "safe" and "more comfortable," which made these women more willing to respond. Blaire illustrates this:

And I would feel more likely to speak up because I know that I would... if they were my friends I would have their support. I wouldn't feel as threatened — but I would also kind of feel a little—-for me, I would feel a little bit obligated to say 
something to set an example. Maybe have other people feel empowered.

Similarly, Paige mentioned:

So I think it would be better in a group of women to say that. That way you feel safer... a group of people to say you know I can make a comment here because if that person's really going to come at me, I have people around that will protect me.

Deter future behavior. While more a motivation for responding to catcalling than a contextual factor, some participants discussed their hope that responding to catcallers could potentially deter their behavior in the future. These women expressed a strong desire to respond to catcalls to discourage men from catcalling other women. Gina described how her response to a group of catcallers could make a difference:

I wish that more women would feel comfortable saying something or responding and kind of my thought and my hope is that me saying something would deter them from doing that in the future to other women I thought you know, if I, if one person says something to them then they might think twice the next time they decided to kind of do that.

As mentioned earlier, Mary was catcalled by group of men who said, "You better not bend over too much otherwise you're just asking for it." She proceeded to respond with "excuse me?" which resulted in the men laughing and scurrying away before Mary could say anything else. Because she had been in an abusive relationship, Mary felt like she "had to say something to them" and thought that "saying something to them definitely made an impact on them, at least I would hope.” 
Maddie expressed wanting to respond to a catcaller in the future:

I think I would try to inform them that "I don't like what you're saying and I don't think that you should be doing that" or I would just. I guess I kind of approach it in the way of like, "Hey stop." I didn't explain why it bothered me, so I think I would try to inform them of why I don't like that rather than just saying like "stop" or "that's annoying."

Maddie then mentioned that catcalling is "a societal problem that doesn't really have a solution," but responding could “change one person's mind."

\section{Factors Influencing Perceptions}

In terms of communicative responses, most women indicated that they ignored catcalls, but they hoped to verbally respond to catcallers in the future. Certain factors influenced women's responses, which demonstrates the importance of examining factors that inform women's perceptions of catcalling. The goal of research question four was to discover the contextual and situational factors that inform women's perceptions of catcalling. Women identified several contextual factors that impacted their view of catcalling: attractiveness, time of day, age, and location. Situational factors such as one catcaller and a group of catcallers also influenced women's perceptions of catcalling.

\section{Contextual Factors}

As previously discussed in the review of literature, Fairchild (2010) determined that contextual factors such as time of day, location, age, and attractiveness were related to women's perceptions of the situation as frightening or enjoyable. Several of my participants identified similar contextual factors that influenced their perceptions of 
catcalling. Time of day was influential as encountering catcalls at night was described as more threatening and frightening, while receiving catcalls during the day was unexpected and even surprising. Women also discussed experiences in a city versus a college town. Receiving comments from catcallers in a city was viewed as more expected but more threatening. On the other hand, in a college town, catcalling was described as expected but less threatening. In terms of attractiveness, some participants viewed catcalling negatively regardless of the attractiveness of the catcaller. Others perceived an attractive catcaller's behaviors as less threatening and less uncomfortable compared to an unattractive catcaller. Another factor, age, coincided with Fairchild's findings, as a younger catcaller's actions were viewed as less threatening and more expected.

Context with alcohol. In contexts where alcohol was involved, women described men's catcalling behaviors as expected, and in a sense, their actions were viewed as less offensive. Several of my participants discussed men's alcohol consumption as a primary factor that influenced their perceptions of catcalling. Women felt as if alcohol consumption was not an excuse for men's catcalling behaviors, but catcalling was described as "more expected" within such contexts. Even though men's behaviors were unjustified, women gave men more leeway as they felt alcohol likely influenced men's behaviors. Lindsey expressed her view of receiving catcalls when going out:

Yeah I mean it can be offensive at a bar or something, but usually you know those people are drinking, and they have a little bit less control over what they're saying, but if you're like in a public place like just a normal every day scene and someone's saying like that saying things like that, that's worse to me that's more 
offensive. And I hate that I'd almost expect it when I'm going out because that's not okay.

Lindsey's experience resonated with participants' perceptions of encountering catcalls in contexts involving alcohol, such as "going out" or "going downtown." Most women reported that catcalling behaviors were more expected in these contexts. As a result, women described "brushing off" catcalls, and catcalling behaviors did not faze them within such contexts. Even though alcohol was involved, women mentioned that alcohol was "not an excuse" for men to engage in catcalling behaviors.

Diana disclosed a specific experience involving two drunken males who catcalled her during the day when she was with her parents:

Actually there was one time when I was downtown with my parents and someone yelled something at me. But I hadn't heard it. My dad did, and my dad was like on his way over to talk to the two males, but I don't know what he said. My dad wouldn't even let me know.

After discussing this experience, Diana mentioned that they were drunk, which was not an excuse for their comments but, "you say things when you're drunk that you obviously don't mean." She then compared her experience to receiving a catcall from someone who is not intoxicated, and felt as if she "brushes it off more" when alcohol is involved.

Time of day. Many of my participants described receiving a catcall at night as more threatening compared to receiving a comment during the day. Experiences at night were associated with feeling "afraid" or "fearful," which echoes Fairchild's (2010) findings as time of day coincided with participants' perceptions of receiving catcalls at 
night as frightening. Some participants disclosed experiences with catcalling that occurred during the day. These were referred to as more surprising and less expected. As previously discussed, Allie's catcalling experience involved a group of men shouting lewd comments to her from their car during the day. She mentioned feeling safer and "preferred that interaction with the cars to happen during the day than at night." But at the same time, Allie was "surprised" that it occurred during the day. She uttered:

I think those things happening during the day shows how pervasive that it is in our culture and in our language, that kind of sexist speech. But at the same time, during the night, it's almost like, more acceptable, because people are trying to party and stuff, whereas during the day, that's who they are during the day. They're not trying to be anybody cooler or have fun that night or anything. Ella expressed similar feelings as she also experienced catcalls from two men during the day:

I was seriously in sweatpants, no make up, and I was walking just outside of Chipotle, the one that's near campus. I can't remember exactly what they said because I just get so uncomfortable. I kind of like freeze up and ignore. It was two guys and they were kind of like snickering and stuff. And they were like, "Hi how are you?" Whatever that's normal. "I'm good how are you?" And they were, "Ha I'm doing good, now" "Oh, I'm doing good, now that you're here, now that I'm seeing you." You know so cheesy, so uncomfortable for me. And then they were just like laughing with each other and like making remarks. I'm just like okay walk away. But I thought it was just a normal interaction. You know, "How are 
you?" "Good how are you? Good?" Oh that was polite. No.

Later on, Ella related back to this experience and she expressed feeling bothered by it because it happened during the day.

Paige described how she felt after an experience in which a man yelled at her during the day as he was driving by:

That shook me up a little bit more even though I was walking to my car. But I remember thinking it's the middle of the day, it's in the middle of a city street, and they weren't under the influence of alcohol. I was still like "so you're going to really take the time to do that?" Who says what you're going to do. So I kind of think that it freaked me out a little bit more. But I think that the daytime helps my perception of it, whereas nighttime I think it's just an easy assumption that nighttime, more shady things could go on. But I don't know. It's like a weird mix that I was quite nervous because the person was not under the influence of alcohol so that was just their own personal stark judgment. But at the same time in the middle of the day in the city street...I was like I don't think anything will happen to me as compared to being in a bar at nighttime and I'm like "OK you never know."

Diana, like several other participants, discussed receiving a catcall at night as more threatening and intimidating because people are normally drinking. Women described feeling "defenseless and afraid," as nighttime is "creepy" and "shady." Diana expressed:

I mean I think like time of day plays a part because you know when it's night out 
you're more susceptible to different activities like I said like drinking and stuff. So I think it could be more threatening that way because like I said people do stupid stuff when they're drunk and when they're impaired I don't know I think it just really depends but maybe more at night just because night's scary.

Blaire mentioned that women are taught to be hypervigilant at night because women are not safe, especially if they are alone at night. She explained:

It's nighttime and we as women have to...instead of taking in the scenery or take our time where we're going it's like I put my keys in between my fingers and hurry it up. And so you don't feel safe to be simply walking down the street in a public space... you shouldn't have to feel that way.

City versus college town. Several participants discussed the difference between experiencing a catcall in the city compared to a mid-sized college town. In a city, being catcalled was identified as threatening and frequent. On the other hand, women described catcalling in a college town as unexpected and less intimidating. Mary explained how the location in which she experiences catcalling affects her perceptions:

I think that a lot of it does have to do with your environment. I mean because I was here on campus, I mean I feel safe here. And I think that if I was in downtown Chicago that's kind of like a sketchy area depending on where you go. I guess it's kind of like a different space. I mean for me it's kind of like it's big, there's a lot of people, and it's busy. So it's if somebody did it there, I would feel more uncomfortable because in situations like that, I would be concerned for my safety. 
Mary then said that she would feel more comfortable in a college town. Even if a lot of people were around, Mary would feel unsafe if she experienced a catcall in a city compared to a college town.

Leslie touched on the frequency of catcalling in a city and particular factors she believed contribute to the frequency:

Now in the city, there's a lot more young professionals... a lot more singles living right down town in the city, and then there's, I don't want to say this, but there tends to be a lot more homeless people, a lot of different populations walking around on the street. I feel like that kind of contributes to the frequency of it happening. Because I know that almost every single time I've walked in downtown Chicago, I've at least gotten one or two comments. I think I worry more about my safety in general in the city than I do in in a college town. Because in a college town, usually its college guys, you know, those type of guys that I don't necessarily worry about as much. But in the city sometimes, you just never know what type of people they are. So, I don't usually comment back in the city. Leslie emphasized that catcalling is a common occurrence in cities, which is a result of the large number of people that live there. Because cities are highly populated, Leslie felt concerned about her safety as she cannot categorize those around her as college students, which contributes to the uncertainty of the context. Leslie feels safer in a college town because she is able to make the assumption that catcallers are college men.

Attractiveness. Another subtheme that arose was that of attractiveness. Several participants felt as if attractiveness did not influence their perceptions of catcalling. Allie 
articulated this:

I mean, the person who says it... I think whatever they say would be rude. I don't think there's a certain kind of person... you know. That there are the good catcallers or the people that are just complimenting you versus the people that are catcalling you. I think if you do it, you're a catcaller. I think if you do it, it's not a compliment. I'm not more open to people saying those things if they look like me or they are my age or my race or something. I don't think it's more acceptable either way.

Some participants viewed attractive catcallers as even less attractive if they engaged in catcalling behaviors. Gina explained:

But I just, I can't imagine, being catcalled even if it were a guy who I thought was physically attractive or you know had feelings for or whatever. I think that if he were to do that, I think that's very unattractive for a man to do that. I can't even think of a situation where I would find that attractive or view that in a positive light.

Gina viewed catcalling in a negative light, and as a result, she did not view an attractive catcaller's comments positively. She actually viewed an attractive catcaller's actions as unattractive.

In accordance with Fairchild's (2010) findings, three participants disclosed that they would view an attractive catcaller's behaviors as less threatening compared to an unattractive catcaller. Halle identified that an unattractive catcaller's actions would make her feel more uncomfortable and fearful compared to an attractive catcaller: 
I think I'd maybe be less inclined to tell him to fuck off if he were more attractive. I'd be like, "thanks." But I think it's different too like when you get, when people catcall you and they're kind of creepy looking, it makes me feel even more uncomfortable. Because you know it's like a cultural thing that when somebody is more attractive you're less inclined to feel weird or you know like someone that looks dirty or something is coming at you, and, you know, people avoid them. I guess it would kind of be like that kind of thing.

Paige also mentioned that her "fear disappears a little bit more" when she gets catcalled by attractive men. She then attributed her perception of attractiveness to "another societal thing that is kind of engrained automatically." Paige described that her fear should not disappear because attractive people are not "above doing harmful things to women," but it is somewhat of an automatic reaction.

Age. Many of my participants expressed that the age of the catcaller affected their perceptions of catcalling as more or less threatening. In accordance with Fairchild's (2010) findings, younger men's catcalling behaviors were viewed as "expected" and less threatening. On the other hand, several women indicated that older men who engaged in catcalling behaviors should "know better," which made their comments more threatening. Halle described how she finds younger men who catcall as less creepy, but older men's behaviors are inappropriate for their age:

I guess like if college guys were doing that to me that doesn't creep me out so much because they're my same age. But it's the older guys who I feel should have more respect for women and who should have learned that these behaviors 
are not appropriate and just through their lives you just...I would assume you know that they have families, they have kids, they have wives, or moms, or sisters. Do they want dirty construction workers yelling at their wives or their daughters? You would think that they grow out of it but apparently not. Paige expressed that when college-aged men catcall, their behaviors are more acceptable because they are "trying to flirt with you." She then stated that by the age of 25 , men should know that it is inappropriate to engage in catcalling behaviors:

I understand that when you're in college that maybe men have not learned life lessons or they're more likely to succumb to social pressure of being a total male an "alpha male" and doing what their friends do even if that might not be what they want to do. I just kind of assume that by the age of 35/40, they understand that they don't have to do that or that they are a fully performing member of society so they should...I assume that those situations should not happen and won't happen outside of people who are essentially my age, like 25 or younger. So when somebody older yells at me...that would freak me out a little bit more because I'm like, 'You're old enough you should know so you really must have these feelings or these thoughts or you have these ideas in your head that you have to make a comment towards me and show me your position in society that you can just say, 'Oh yeah you're looking hot.'”

\section{Situational Factors}

Some participants discussed situational factors that influenced their perceptions of catcalling. Women identified one of the factors as a group of men. If men were in a group 
and engaged in catcalling behaviors, women perceived the behaviors as offensive but less threatening because their behaviors resulted from group members "egging each other on." This connects with Pryor, LaVite, and Stoller's (1993) description of group norms as the composition of a group creates a sense of anonymity and increases men's likelihood of engaging in harassing behaviors. Women emphasized that men would feed off of one another when in a group and continue engaging in catcalling behaviors. The second factor when one man engaged in catcalling behaviors, women described it as "more threatening" and "scarier" because the catcaller's behaviors could escalate and result in an attack.

Group norms. Several participants discussed receiving catcalls from a group of men. One man would make a comment, the rest would "join in," and the men would continue to "egg each other on." Women perceived these behaviors as offensive and a result of men trying to impress their friends. When Mary dropped something on the ground, as noted above, and proceeded to pick it up, the comments were from three males. She expressed her perceptions in regards to receiving catcalls from a group of men:

I think I would have felt more upset because it wasn't just one. It was like they all had the same thought and made little snickers and stuff. There was the one guy who said that comment, and then the other two said little things like um, just like stupid little comments like, "Yeah, that's too short of a skirt." Just stupid stuff like that. And I think that maybe if it was one person, it wouldn't have affected me as much. But because it was like three different people and three different 
minds like thinking the same thing, that it was okay to say those things, kind of affected me as opposed to if it was one.

Gina described receiving catcalls from a group of men as less threatening because men engage in such behaviors to adhere to social pressures:

Usually if it's a group of people it's less threatening because it's just like one guy saying something and then everyone like laughing and trying to look cool and yell stuff at you. So I know that usually with a group it's just words.

One catcaller and escalation. Some women described receiving a catcall from one man as threatening and intimidating because less people were around to intervene if the situation escalated. Allie explained her perception of one catcaller compared to a group of catcallers:

I think it's more alarming when it's one person alone and away because there is less of an audience. And if they wanted to attack you, they probably could. If it is one lone person, saying weird stuff to you, I think it would be more likely that an attack would happen than in a group.

Allie felt as if situations involving one catcaller could escalate because the catcaller was not in the presence of other men who might catcall as well. Allie then said, "It's more likely that it's a threat if it's one person" as one catcaller could attack without others intervening.

Halle discussed a hypothetical situation in which she received a catcall from one man. She had normally experienced catcalling when men were surrounded by other group 
members. Halle expressed that she would feel threatened if she received a catcall from one man:

Yeah, if I was alone and somebody was like catcalling me down the street, I would feel really threatened. I would definitely want to stay in somewhere that was public just because you know you don't...you don't know what people are going to be doing. You know people will follow you.

Riley described situations with one catcaller as more alarming:

Yeah, that has happened before, a few times - not as common-it's a lot scarier when it's one person that's does it, which doesn't really make sense but then if it's just like one person just kind of like tailing behind you like yelling stuff...if it's just one person like they're not doing it to impress anyone they're literally just trying to get to you and like follow you.

\section{Women's Experiences as Trivialized}

Both women's responses to catcalling and the immediate factors that impacted both their perceptions and their responses are situated within a larger social context - the perception that women's experiences of catcalling are trivialized. Some women conveyed various ways in which their catcalling experiences were minimalized. These women declared that men and even other women often failed to acknowledge the effects that catcalling has by describing catcalling as complimentary. Women also emphasized that the media downplay the effects of catcalling by portraying catcalling as funny or showing that women enjoy receiving catcalls. 


\section{Minimizing Catcalling Behaviors}

Failure to acknowledge effects. Some of the women expressed that men failed to acknowledge the severity of their behaviors as they would "laugh it off" and describe catcalling as complimentary. Other women would brush off men's behaviors as the norm because catcalling is commonplace. Lindsey explained how men fail to take catcalling seriously because they view it as complimentary:

It really shows the dominance men still have over women. As much as I don't like that, I wish they would take it more seriously, like I wish men would recognize that it is an issue. They make comments about women just being like, "Oh [I] can't give a girl compliment." You're not giving her a compliment—you're yelling across the street or across the room at her saying something rude. And if she takes it offensively, that's offensive. There's no other way around that. They make it minimalized, the issues. I just I really wish that more guys would turn to another guy and say, "That's not okay" instead of us having to defend ourselves all the time. Just recognize that that is not okay. It's okay to say that's not okay. Maddie described how men typically brush off their actions in a joking manner: I think they just kind of usually brush it off just like...I feel like they're just like, “Oh I'm just kidding” or like—you know the guy will just be like, “Oh I don’t— all that—I didn't really mean it." It's like, "Yes you did. You clearly meant to say that." 
Maddie then discussed that men are not self-aware of "what their actions do." She expressed that they just think it is funny and are "not self-aware of how that may make someone feel," which minimalizes the effects that catcalling has on women. Riley mentioned that men view catcalling as complimentary and funny: There's always been a few people who are like, "Oh you should take it as a compliment," and "I'm like no it's really not" stuff like that. It's always boys that say that too. Even like some of my guy friends that I've talked to-like I'll bring it up, and they'll be like, "Oh it's not that big of a deal.” They just don't think that it happens that often or they don't realize like if they say one thing to a girl one night like, "Oh it's not a big deal like I just said one thing." But that probably happened to that girl ten times in one night because that's happened. Like one guy will say one thing to you but then you encounter like, you go out downtown and ten guys in one night are all yelling random stuff at you, so I think they just need to be more educated on it, just educated on just women in general probably. Because they just don't see it as threatening. They just think they're being funny and they don't see how it could be taken in such like a scary way. Mary expressed that some women also fail to recognize the severity of catcalling, which minimalizes men's behaviors and how they affect women: My friends would say "Mary you know it's not that big of a deal, it's fine. It's whatever they were just messing around.” And I guess I get very passionate about it, you know you're women and you're saying that they're just messing around. That's the same as saying boys are just boys. Just because it's messing around 
doesn't mean that it's not hurtful and it doesn't mean that it's okay. And I think that a lot of the time, women don't take it as seriously as it is.

How the media portray catcalling. Some participants discussed how the media's portrayal of catcalling minimalizes catcalling as a serious issue. Specifically, women mentioned that catcalling is depicted as "funny" and women are often "happy" when receiving catcalls. Mary expressed how the media impact the way in which society treats women and views issues like catcalling:

I think that the media has a lot to do with it just because of what I mentioned before about how the boys will be boys. This whole culture of how we think about women and how men treat women has definitely been twisted and turned in social media, and I say this generation is so media based that that has a lot to do with how we think about situations like this.

Lindsey discussed the media's impact as well as music videos' unrealistic portrayal of women's approval of catcalling behaviors:

We're surrounded by influences, and it's probably a lot of things that I don't even realize. I'm sure that subconsciously the media kind of instilled in my mind what is positive or negative. Yeah, and then in music videos, they'll show women that are just like these guys could do and say whatever they want to them. They're just so happy about it—well no. I'm not happy about it, that's not realistic. You can't take what is being said in a rap song and apply it to real life and expect it to be transparent because it's not, it's not at all.

Diana mentioned that women do not view catcalling as a serious issue: 
I mean when you see it in like T.V. shows you know girls like laugh it off and stuff, and, I mean, I don't think it's a funny thing. And I even see you like some of my friends or like if that happens to someone they just kind of like laugh and stuff as if it's a compliment. But I think the media kind of makes it like not like a joke but like you see girls take it more as a joke than a more of a like a serious thing.

\section{Conclusion}

Based on the transcriptions and analysis, it is evident that catcalling is a complex issue. Catcalling was defined as a man making sexual or derogatory comments towards women; however, some women emphasized that it often involved nonverbal behaviors. Catcalling was conceptualized as a primarily negative experience, but non-descriptive comments such as "you look good" were described as positive. Even if women viewed certain comments positively, they never described catcalling as complimentary; rather, the women felt that men perceived their own comments to be compliments. As a result, women's perceptions of catcalling are devalued as men, and society as a whole, fail to recognize the implications of men's catcalling behaviors. Catcalling was associated with negative emotions and affective states, which demonstrates the negative effects of catcalling. Women communicatively responded to catcallers by avoiding, ignoring, or making verbal comments in hopes of combatting the negative effects, but regardless of women's responses, they continued to feel threatened and offended. These findings reveal much greater depth behind women's catcalling experiences, which reflects these women's standpoints. Women's ability to communicate their experiences and perceptions of catcalling allows them to critically reflect on men's catcalling behaviors. In the 
subsequent chapter, I will review emergent themes, as well as provide practical and theoretical implications for future research. 


\section{CHAPTER V \\ DISCUSSION}

The primary objective of this study was to give voice to women who have experienced catcalling, while simultaneously contributing to the lack of research on a topic that continues to be trivialized. Based on the scarcity of research examining women's catcalling experiences, there are several components within this topic of interpersonal and feminist research that should be explored further. In particular, this study helps to start a conversation between academics and social activist groups regarding women's standpoints on catcalling.

Since my research emphasized women's personal experiences as well as their perceptions of catcalling, I felt it was essential to conduct respondent interviews to obtain detailed responses. This qualitative method of data collection allowed participants to express their experiences and views. Therefore, I conducted 13 interviews with collegeaged women from small or midsized communities who had experienced catcalling at some point in their lives. By using qualitative research, I was able to gain an understanding of women's narratives and perspectives as each woman expressed a different story. In this chapter, I will first provide more detailed interpretations of participants' responses to the research questions. Then, I will discuss the strengths and limitations of this study, and lastly, I will evaluate opportunities for future research. 


\section{Discussion of Research Questions}

\section{"It's Not a Compliment but..."}

In response to research question one, women provided their own definitions of catcalling. Participants' definitions often varied in terms of the behaviors that constitute catcalling. Nine participants identified catcalls as verbal utterances, while four participants identified catcalls as involving both verbal and nonverbal behaviors. Even though women's definitions varied in regards to the types of behaviors that catcalling is comprised of, all 13 participants described catcalling as a man yelling sexual or derogatory comments towards a woman. This connects with Chhun's (2011) identification that catcalling involves the use of "crude language." Because women defined catcalling in this manner, it is clear as to why 11 of 13 participants indicated that they did not view catcalling as a compliment; rather, they felt men view their own comments as compliments. Paige and Blaire emphasized that men often perceive their comments as a means of complimenting a woman because their comments highlight a woman's beauty and physical appearance. Participants went on to discuss different strategies men could employ to actually compliment a woman, such as Mary's description of walking up to a woman and complimenting her during a conversation. Women's indication that catcalling is not a compliment, and their suggestion of potential strategies to compliment women, coincides with Gardner's (1980) argument that comments from strangers in public places violate the norms of complimentary behaviors. In the perception of most participants, proper comments are not given by male strangers in public places. 
As Goffman (1963) asserted, strangers tend to ignore one another in public places with the exception of brief eye contact, which is known as civil inattention. Catcallers' comments are not received as compliments because men symbolically force themselves into a woman's personal space and breach the notion of civil inattention. Twelve of the 13 participants discussed the notion of "it was a stranger" as a major contributing factor towards viewing catcalling behaviors as negative and offensive rather than complimentary. Men are thus treating women as open persons in public places, which coincides with Tuerkheimer's (1997) assertion that men utilize breaches of civil inattention to make comments towards women, thus defining women as objects. Catcalling was not only identified as an inappropriate way to compliment a woman, but several women expressed that catcalling is a means of objectifying them. This reaffirms Fredrickson and Robert's (1997) objectification theory as catcalling functions to treat women as bodies or objects to be consumed by others. Even though most women indicated that they did not view catcalling as complimentary, Paige, Leslie, and Lindsay explained that it felt good to be noticed, and catcalling often provides a boost of confidence. Paige specifically mentioned that, at first, catcalling made her feel glad that she was attractive, but then a few seconds later, she described feeling objectified. This reinforces the notion that women are primarily valued for their bodies and nothing else, so they must then focus on their outer appearance in order to be deemed valuable by society. Fredrickson and Roberts assert that women are constantly portrayed as objects via various forms of media such as: advertisements, music videos, and pornography. It is almost impossible to avoid sexualized images of women's bodies in 
American culture, which demonstrates that "the sexual objectification of the female body has clearly permeated our cultural milieu" (p. 177). As a result, women are taught that their bodies are constantly being evaluated and objectified.

Based on the transcribed data, catcalling functions as a means of sexual objectification. Therefore, catcalling, like many other forms of objectification, reminds women that they are on display, which leads to women's continued preoccupation with their outer appearance.

\section{"I Felt..."}

The responses in accordance with research question two revealed the emotions and affective states participants felt after being catcalled. Specifically, participants' responses indicated primarily negative emotions and affective states. Participants did not express positive emotions or affective states, such as happiness or gratitude, which emphasizes the emotional harm that catcalling inflicts on its victims. Tuerkheimer (1997) asserted that some forms of harassment leave women's bodies physically untouched, but when women experience them, "deep harm has nonetheless been inflicted" (p. 190). This relates to catcalling as participants indicated that catcallers never engaged in physical contact, but men would yell comments that were often rude and derogatory. In turn, women felt angry, annoyed, uncomfortable, nervous, and embarrassed.

The findings suggest that women are negatively affected by catcalls as such remarks trigger negative emotions and affective states that impact women's emotional health and well-being. The emotions and affective states women identified emphasize women's desire to minimize the harms catcalling induces as well as the desire to freely 
move about a public space without feeling constrained. Bowman (1993) found that street harassment elicits feelings of anger, humiliation, and fear. Similarly, catcalling evokes anger, embarrassment, and nervousness. Catcalling thus causes women to suffer "a psychological beating in the form of emotional distress and feelings of disempowerment" (p. 537). Halle was one of several participants who expressed that catcalling is commonplace, something that women start experiencing when they are young. As a result, women have likely experienced various negative emotions and affective states for quite some time, which indicates that women have constantly undergone emotional trauma.

\section{"It's a Lose-Lose for Women"}

Research question three established that women utilize various response strategies when encountering men's catcalling behaviors. Participants made choices to ignore, verbally respond, or avoid catcallers all together. Certain factors such as the catcaller's comments moving from "positive" to offensive impacted the response strategies participants utilized. Some women described responding to catcalls as "lose-lose" because, regardless of whether a woman ignores the behavior or verbally responds, catcalling often leads to escalation. Halle mentioned ignoring catcalls to prevent showing catcallers she was annoyed or bothered by their behaviors, which connects to Bowman's (1993) assertion that women ignore street harassers to "mask feelings of invasion, anger, humiliation, and fear they suffer" (p. 537). Halle ignores catcalls to mask her feelings, while simultaneously attempting to prevent escalation, but even ignoring catcalls can lead to their escalation. 
Women who respond also risk the chance that the situation will escalate and become threatening. Only three participants, Gina, Halle, and Leslie, reported verbally responding to catcallers. Halle expressed that verbally responding to catcalls often resulted in escalation and remarks like "bitch." As Kearl (2010) demonstrates, responding to street harassers can escalate into threatening situations, even violence or murder. Participants' communicative responses to catcallers thus indicate that "women can't win." Refusing to respond to catcaller's can result in more hostility; however, verbally responding can also result in life-threatening situations (Chhun, 2011). The analysis of participants' responses makes it clear that, regardless of women's response strategies, they "emerge from the encounter with an overwhelming sense of disempowerment" (Tuerkheimer, 1991, p. 191). Women lose with either strategy as they risk escalation and often walk away feeling disempowered.

Despite the danger of escalation, women often verbally respond to catcallers, which emphasizes participants' awareness of how men infringe on their mobility in public spaces. According to Wood (1994), "It is through discursive activities that individuals and groups critique existing ideologies and author oppositional ones ... changing cultural understandings requires disrupting dominant discourses and the ideologies they embody' (p. 26). Responding to catcallers communicates women's oppositional standpoints to the catcallers with whom they come in contact. In a way, women's communicative responses disrupt societal discourses that define catcalling as a means of complimenting a woman, contrary to Glamour magazine's 1969 advice about how to properly respond to men's verbal comments in public, noted in the review of 
literature. Because women respond to catcalls, they are now actively resisting such discourses, which reveal how standpoints shape their unique knowledge, leading to resistance through their communicative responses.

\section{"Halo Effect"}

Regarding research question four, responses revealed that certain contextual and situational factors inform women's perceptions of catcalling. One theme, attractiveness, demonstrates the complexity of catcalling. Two participants described that the attractiveness of the catcaller did not alter their view of catcalling. Allie and Gina asserted that an attractive catcaller's actions are not viewed as more acceptable than an unattractive catcaller's. On the other hand, Halle and Paige's responses coincide with Fairchild's (2010) findings. She determined that contextual factors such as attractiveness altered participants' perceptions of stranger harassers. An attractive perpetrator's actions were viewed as less threatening compared to an unattractive perpetrator. Halle and Paige described that they would view an attractive catcaller's actions as less threatening and feel less fearful of his behaviors. Halle referred to this notion as "a cultural thing that when somebody is more attractive you're less inclined to feel weird." Paige also mentioned that perception of attractiveness is "another societal thing that is kind of engrained automatically."

Paige and Halle's perception of attractiveness connects to the concept of the "halo effect," which asserts that "individuals who are attractive receive the benefit" of the effect (Fairchild, 2010, p. 195). That is, an attractive individual is often given more leeway because people associate additional good qualities with attractive individuals per 
the attractiveness stereotype. Golden, Johnson, and Lopez (2001) conducted a study that examined the effects of attractiveness on perceptions of sexually harassing behaviors in the workplace. The researchers found that participants viewed attractive male perpetrators' harassing behaviors as less threatening compared to unattractive perpetrators. Participants' perceptions of sexual harassment stemmed from the stereotype of attractiveness or the "halo effect." My findings indicate that some participants exhibited the "halo effect" in terms of perceiving an attractive catcaller's behaviors as less threatening. Thus, contextual factors such as attractiveness influence women's perceptions of the severity of catcalling behaviors.

Based on findings in regards to contextual factors such as "context with alcohol," "time of day," and "age," women's expectations were often violated. Several participants described the unexpected nature of receiving catcalls during the day. On the other hand, receiving catcalls at night was identified as expected especially if alcohol was involved. In terms of age, if older men engaged in catcalling behaviors, women described the behaviors as unexpected compared to younger men. Burgoon's expectancy violations theory (EVT) is especially applicable when studying women's perceptions of catcalling. EVT proposes that our expectations are influenced by "the communicator, the relationship, and the context in which the interaction occurs" (White, 2015, p. 219). Contextual elements such as how an individual should communicate in a specific setting influence a communicator's expectations of the interaction.

When an expectation is violated, individuals make sense of what is happening by focusing on the violating behavior. As a result, arousal is heightened, which initiates 
cognitive appraisals. These appraisals involve an individual determining the meaning of the behavior and then evaluating the violation positively or negatively, which is known as violation valence. The reward value of the violation influences the valence of the violation (White, 2015, p. 220). That is, if an individual is viewed as physically attractive, powerful, or competent, he or she is seen as more rewarding compared to an individual who does not maintain any of those characteristics. When a rewarding communicator violates the expectations of their interaction partner, the partner is more likely to evaluate the violation positively. As the findings demonstrate, some women indicated that they viewed an attractive catcaller's behaviors as less threatening. In accordance with EVT, attractive catcallers' behaviors were viewed as more rewarding, and in turn, women evaluated the violations positively.

While it appears that EVT is applicable to studies involving catcalling, researchers must consider expanding upon the theory in order to apply it to future studies. The findings demonstrate that women do not simply evaluate a catcaller's behavior or assess the catcaller's reward valence. Women also evaluate contextual factors such as age and location in terms of expectancy violations. When receiving catcalls from older men, many participants perceived the comments as more threatening and less expected because men at that age should "know better." Level of threat and expectancy are thus connected as catcallers who pose greater threat (i.e., older men) negatively violate women's expectations. Younger men who engage in catcalling behaviors were viewed as less threatening, indicating a positive violation of women's expectancies. Location plays a similar role as participants described receiving catcalls in a college town as unexpected 
but less threatening (i.e., positive violation). This has implications for women who experience catcalling in college towns. Positive violations likely reinforce society's conceptualization of catcalling as complimentary and a less serious issue. Therefore, the application of EVT is essential for future research as contextual factors impact how women evaluate catcalling in terms of a violation of their expectations.

\section{"Catcalling Isn't a Serious Issue"}

The responses in relation to research question five revealed that women's catcalling experiences are often disregarded as men fail to recognize the effects of their actions, women brush off catcalling as "not that big of a deal," and the media portray catcalling as funny. Riley and Lindsey emphasized how men fail to recognize catcalling as a serious issue because they think that it's funny or that women "should take it as a compliment." Men thus minimize catcalling by saying that it "isn't a big deal" and that catcalls function to compliment women. Chhun (2011) indicated that "some men believe the purpose of the verbal act is to compliment" (p. 280). My participants felt that men continue to view catcalling as complimentary, which is problematic as catcalling makes women feel objectified, degraded, and threatened. This demonstrates the dominant group's attempt to discursively structure women's perceptions of catcalling. Men's identification of catcalling as complimentary strengthens the connection between the constraints placed on women's perceptions by dominant groups and the creation of unique feminist standpoints.

Participants also indicated that the media portray catcalling as a trivial issue. Women mentioned music videos, rap songs, and T.V. as media through which catcalling 
is portrayed as funny. Diana discussed that the "media kind of makes it like not like a joke but like you see girls take it more as a joke than a more of a like a serious thing." This demonstrates that women are taught to view catcalling as complimentary through different forms of media, which coincides with Fairchild's (2010) assertion that women are socially conditioned to view men's harassing behaviors as complimentary. Based on the transcribed data, women's concern and disapproval of catcalling continues to be silenced as a result of men's view of catcalling as complimentary, as well as the media's portrayal of catcalling as a trivial issue.

In this study, the women recognize that dominant discourses contradict their own experiences and perceptions of catcalling. According to Hartsock (1983), recognizing the inaccuracies of the dominant group's prescriptions of oneself molds a feminist standpoint. From their positions in society, women "see both how their culture tells them what it means to be female and how their own experiences of being female often do not mesh with these cultural prescriptions''(Hallstein, 1999, p. 34). Women's distrust of dominant discourses that devalue their experiences reveals their resistance of the constraints placed on them to view catcalling as complimentary and inoffensive. By recognizing and challenging how society views catcalling, women are able to construct the meaning of catcalling that correlates with their own standpoints.

\section{Strengths, Limitations, and Directions for Future Research}

\section{Strengths and Limitations}

The first strength of this study involves the 13 interviews I conducted, which is a sufficient sample as I reached redundancy after the first 9 interviews. I reached 
redundancy when no new categories emerged from the data (Lindlof \& Taylor, 2011). I continued collecting interviews to give voice to those who had contacted me about participating in my study. This both established that I had sufficient exposure to participants' comments and understandings (Fitch, 1994) and provided uncoded data to compare to my existing categories, fulfilling the function of "referential adequacy," a tool for determine the trustworthiness of the conclusions (Lincoln \& Guba, 1985). In addition, this study was unique as participants were recruited from small or midsized Midwestern communities. Studies regarding street harassment have primarily focused on urban areas. As street harassment often forces women to change their routes in order to avoid harassers, Bowman (1993) indicates that in doing so, women are relegated to the private sphere. She identifies that urban environments become uncomfortable for women as street harassment reminds women that they are not welcome in the public sphere. Benard and Schlaffer's (1984) empirical study also indicated that street harassment takes place in larger urban areas such as New York City, and that it is uncommon in suburban areas. Thus, the present study is unique because the findings indicate that women do in fact experience harassment, more specifically catcalling, in suburban areas. This demonstrates that catcalling is a widespread harm as it is not solely confined to urban areas. Women can experience catcalling anywhere, at any time, which makes it difficult to avoid. Thus, catcalling is a serious issue as women encounter the harm in both suburban and urban areas. As the current study interviewed college-aged women from communities dominated by college populations, future research should examine women's catcalling experiences outside of college towns. It is likely that receiving catcalls in suburban 
communities without the presence of college populations would be viewed very differently. As several of the participants emphasized, receiving catcalls in a college town was viewed as less threatening because catcallers were normally college males. Outside of college towns, women are unable to attribute catcalling behaviors to college-aged males, which likely impacts women's perceptions of catcalling. A clear strength involves the use of respondent interviews. Such interviews allow participants to express their experiences and perceptions in an open-ended manner. That is, participants were able disclose their subjective standpoints on catcalling, which allowed them to voice their standpoints in their own words. Respondent interviews also provided participants with the ability to discuss their experiences in the form of narratives. By utilizing respondent interviews, I was able to capture participants' life experiences and the meaning they placed on their experiences in their own language (Lindlof \& Taylor, 2011).

Although this study had several strengths, there were also clear limitations. First, as a feminist woman who has experienced catcalling and views men's catcalling behaviors as offensive, it is possible that I may consciously or subconsciously emphasize certain responses or results and deemphasize others. My presence as an interviewer may yield different responses compared to someone with different views of catcalling. On the other hand, my knowledge as someone who lives a woman's standpoint may increase the credibility of the analysis as I recognized the individuality of women's standpoints and supported my analysis with a multitude of direct quotations from participants. The second limitation involves the participant selection process. As I utilized a convenience sample, 
participants were self-selected into this study. It is likely that those who were motivated to disclose their experiences actively volunteered.

An additional limitation is that participants relied on recall of experiences, some of which had occurred a few years prior to the interview. Participants may have been unable to recall minute details that were actually quite significant. Research could be conducted in which participants utilize reflective journals, which would allow participants to describe their perceptions or emotional responses quickly after an experience with catcalling. Finally, the limitation of the sample suggests the need for a more diverse sample. Although the focus of this study did not have much to do with participants' age, some participants indicated that they viewed catcalling as more enjoyable when they were younger. This signifies the need for a sample with a wider age range. Younger teens and older women might perceive catcalling differently and express unique standpoints compared to college-aged females. Paige discussed an experience in which she was 16 and received catcalls from men. She described feeling nervous and afraid in response to that specific experience. Thus, teenage girls who start experiencing catcalling at a young age are likely to have different standpoints compared those who start receiving catcalls when they are adults. In doing so, researchers would be able to determine if, and how, receiving catcalls at a particular age impacts women's perceptions of catcalling.

\section{Future Research}

There are a number of avenues for future research that scholars should explore in regards to catcalling. First, although catcalling has rarely been examined as a separate form of harassment, looking at the differences between perceptions of women who are in 
long-term committed relationships compared to participants who identify as single may provide researchers with valuable results. New research may suggest that women who are in committed relationships brush off catcalls more than single women because they feel secure in their own relationships. Single women may view catcalling as a boost of selfesteem or confidence as such remarks may be perceived as less offensive. It is also necessary for researchers to explore sexuality and the role that it plays in terms of how lesbians view catcalling. Kearl (2010) indicates that men who street harass lesbian women often reference their sexual orientation and they do so with hostility. Thus, these women may receive catcalls that differ from those that heterosexual women receive. As a result, lesbian women likely have different standpoints that develop from their experiences and perspectives, which stem from their positions in society as lesbian women. By exploring lesbians' perceptions of catcalling, researchers may call into question the cultural norm that catcalling is heterosexual and involves "heterosexual" men catcalling "heterosexual" women.

Additionally, researchers may benefit from assessing men's perceptions of catcalling as well as their motivations for engaging in catcalling behaviors. Feminist theories are grounded in the lived experiences of individuals who are often deemed subordinate. Feminist scholars are concerned with "treating gendered, sexual, racial and ethnic cultures and their experiences as serious and important" (Rakow \& Wackwitz, 2004, p. 6). That being said, feminist scholars tend to focus on the experiences of women in order to evoke change. In doing so, they rarely examine men's understandings and conceptualizations of the world. It is necessary to examine men's motivations to engage 
in catcalling behaviors in hopes of generating new ideas about catcalling. This could potentially encourage men to rethink the enactment of catcalling behaviors, which may decrease the prevalence of catcalling. It may also provide scholars with the opportunity to educate those who believe catcalling affects victims in a positive manner. My participants often discussed the need for men to be educated about catcalling and the effects it has on women. Riley expressed that men need to be educated about catcalling "because they just don't see it as threatening. They just think they're being funny and they don't see how it could be taken in such like a scary way." By conducting a qualitative study that examines men's perceptions of catcalling, researchers may bring about new understandings of catcalling. In turn, men, and society as a whole, may become more aware of the negative effects that catcalling has on women.

Not only should communication scholars conduct studies with men in regards to catcalling, but researchers may also consider focusing on the emotions and affective states that women express after being catcalled. This study indicates the importance of examining women's emotional responses to catcalling as participants expressed negative emotions or affective states. By conducting a quantitative study that primarily focuses on emotions, researchers may be able to reach more generalizable conclusions about the emotions women express in response to catcalling. A quantitative study may also be beneficial in terms of examining the emotions and affective states that women express before, during, and after receiving a catcall. It is possible that participants may feel different emotions or affective states during the catcalling episode compared to after. That is, a woman may feel the emotion "anger" when she receives a catcall, but that 
anger may lessen and result in the less intense affective state of "annoyed." Or she might feel an initial sense of pride, especially if she has already prepared herself to look nice or feels confidence in her appearance, but that may turn to disgust or annoyance as the catcalling leaves a bitter emotional aftertaste. Future quantitative investigations could provide researchers with a more comprehensive understanding of the ways and degrees to which women are affected by catcalling.

This study also indicated that the media likely influence women's perceptions of catcalling. Future researchers may conduct focus groups to allow individuals to talk freely about their viewpoints (Lindlof and Taylor, 2011). Using focus groups could prove particularly useful because this method allows participants to bounce ideas off of one another and have an in-depth discussion about their views. As participants described, the media's portrayal of catcalling contradicts most women's perceptions. Researchers may benefit from using focus groups as the setting will likely allow participants to discuss the contradictory discourses of catcalling that exist in our society. Also, as a result of the media's indication that catcalling functions as a means of complimenting a woman, it is noteworthy to conduct a study that explores how women who have never experienced catcalling view it. It is possible that these women may feel inadequate because they have not experienced catcalling. Because our culture so often objectifies women and illustrates that women are valued primarily for their bodies, these women may feel inferior to women who receive catcalls as well as maintain different standpoints compared to such women. 
While the topic of catcalling is highly under-researched, the exploration of women from specific ethnic backgrounds is also necessary. A clear limitation of the current study is that a majority of participants were Caucasian. Researchers should consider examining women of various ethnicities because it is possible that they experience catcalling differently. Women whose family members grew up outside of the United States may perceive catcalling as a means of gender discrimination. Oftentimes, catcalling stems from traditional gender norms in societies where women continue to maintain marginalized positions. In countries such as Iran, women are essentially punished for stepping outside traditional boundaries. Various cultures have "strong traditional rules that people, especially women, are supposed to follow" (Lahsaeizadeh \& Yousefinejad, 2011, p. 18). Women's bodies are then exploited in various ways such as catcalling, which reinforces the spatial boundaries that encourage traditional values. Thus, it is imperative for scholars to conduct research pertaining to women from various ethnic backgrounds. In doing so, scholars may develop a better understanding of how women perceive catcalling and the factors that influence perceptions.

\section{Summary}

This study demonstrates the importance of using qualitative interviews to understand the depth of women's experiences and the knowledge that comes from women's standpoints. Not only does this qualitative project provide new information about an under-researched topic, this examination gives voice to women and their experiences with catcalling. Participants' subjective standpoints will provide researchers 
within the fields of interpersonal, feminist, and women and gender studies valuable information regarding catcalling.

Based on my personal preconceptions and experiences, I had assumed that women would view catcalling negatively; however, respondent interviews demonstrated that women view some catcalls as providing a boost of confidence. It appears that our society has emphasized that women need their beauty reaffirmed by others. As a result, catcalling functions as a means in which women are sexually objectified. The consequence of sexual objectification is that women begin to treat themselves as objects. While previous research indicates that street harassment is a means of sexually objectifying a woman, it is difficult to assume the same for catcalling based on the scarcity of research. It is vital for researchers to examine this topic as it is under-researched and results in negative effects such as women's self-objectification. It is also important for researchers to continue examining the relationship between sexual objectification and catcalling because it appears that some women evaluate their catcalling experiences positively. Previous research fails to make the connection between the positive effects of objectification and catcalling. Because Fredrickson and Roberts' (1997) objectification theory accounts for the positive effects of sexual objectification, researchers should continue exploring the topic of catcalling in hopes of extending the theory to include catcalling and how it often functions a positive experience for women.

This project indicates that women's catcalling experiences are trivialized as their perceptions of catcalling diverge from society's and men's conceptualization of catcalling as complimentary. It is my hope that this study will encourage women to communicate 
their experiences with others as well as communicate the need for catcalling to be considered a serious issue. It is disheartening that women feel as if they "can't win" when it comes to catcalling. This signifies the importance for scholars to continue examining catcalling as a lot of progress needs to be made in terms of creating an understanding of catcalling as harmful. Even though catcalling was described as a "lose-lose" for women in terms of communicatively responding to catcallers, the continued study of this particular topic will likely create resources for women that will help them communicate their experiences with other women while determining the best way to respond to catcallers. Although creating resources may be beneficial, the media need to portray catcalling as a serious issue rather than depicting it as funny or showing that women enjoy it. As a result, women may feel more apt to discuss their experiences with family, friends, and romantic partners.

Scholars' continued study of this topic will likely educate men and society as a whole about the effects of catcalling, which could potentially deter men from engaging in catcalling behaviors and encourage society to view catcalling as a serious issue. As someone who has personally experienced catcalling, it is my hope that this study will bring to light the importance of educating women and men about this issue. Education and communication could make a difference in terms of framing catcalling as potentially harmful. As a result of bringing this topic into schools and communicating about how catcalling affects women, our society might be one step closer to a culture in which women no longer experience men's evaluative comments in public spaces. 


\section{REFERENCES}

Bartky, S. L. (1990). Femininity and domination: Studies in the phenomenology of oppression. New York, NY: Routledge.

Bates, L. (2013, April 16). The everyday sexism project: A year of shouting back. TheGuardian.com. Retrieved from http://www.theguardian.com/lifeandstyle/thewomens-blog-with-jane-martinson/2013/apr/16/everyday-sexism-projectshouting-back

Baxter, L. A. (1991). Content analysis. In B. M. Montgomery \& S. Duck (Eds.), Studying interpersonal communication (pp. 239-254). New York, NY: Guilford Press.

Benard C., \& Schlaffer E. (1984). The man in the street: Why he harasses. In A. M. Jaggar \& P. S. Rothenberg (Eds.), Feminist frameworks (pp. 395-398). New York, NY: McGraw-Hill.

Berdahl, J. L. (2007). The sexual harassment of uppity women. Journal of Applied Psychology, 92, 425-437. doi: 10.1037/e633962013-260

Blumenthal, J. A. (1998). The reasonable woman standard: A meta-analytic review of gender differences in perceptions of sexual harassment. Law and Human Behavior, 22, 33-57. doi: 10.1023/A:1025724721559

Bowman, C. G. (1993). Street harassment and the informal ghettoization of women. Harvard Law Review, 106, 517-580. doi: 10.2307/1341656

Braun, V., \& Clarke, V. (2006). Using thematic analysis in psychology. Qualitative Research in Psychology, 3, 77-101. doi:10.1191/1478088706qp063oa

Celik, Y., \& Çelik, S. Ş. (2007). Sexual harassment against nurses in Turkey. Journal of Nursing Scholarship, 39, 200-206. doi: 10.1111/j.1547-5069.2007.00168.x

Chhun, B. (2011). Catcalls: Protected speech or fighting words. Thomas Jefferson Law Review, 33, 273-295. 
Darley, J. M., \& Latané, B. (1968). Bystander intervention in emergencies: Diffusion of responsibility. Journal of Personality and Social Psychology, 8, 377-383. doi: $10.1037 / \mathrm{h} 0025589$

Davis, D. E. (1994). The harm that has no name: Street harassment, embodiment, and African American women. UCLA Women's Law Journal, 4, 133-178.

Dougherty, D. S. (2001). Sexual harassment as [dys]functional process: A feminist standpoint analysis. Journal of Applied Communication Research, 29, 372-402. doi:10.1080/00909880128116

Fairchild, K. (2010). Context effects on women's perceptions of stranger harassment. Sexuality and Culture, 14, 191-216. doi:10.1007/s12119-010-9070-1

Fairchild, K., \& Rudman, L. A. (2008). Everyday stranger harassment and women 's objectification. Social Justice Research, 21, 338-357. doi: 10.1007/s11211-0080073-0

Feis, A. (2014, October 8). Woman's throat slashed after rejecting man's advances. NYCPost.com. Retrieved from http://nypost.com/2014/10/08/womans-throatslashed-after-rejecting-mans-advances/

Firestone, J. M., \& Harris, R. J. (1999). Changes in patterns of sexual harassment in the US Military: A comparison of the 1988 and 1995 DoD surveys. Armed Forces \& Society, 25, 613-632. doi: 10.1177/0095327x9902500405

Fitch, K. L. (1994). Criteria for evidence in qualitative research. Western Journal of Communication, 58, 32-38.

Foss, K. A., \& Foss, S. K. (1994). Personal experience as evidence in feminist scholarship. Western Journal of Communication, 58, 39-43. doi: 10.1080/10570319409374482

Fredrickson, B., \& Roberts, T. (1997). Objectification theory: Toward understanding women's lived experiences and mental health risks. Psychology of Women Quarterly, 21, 173-206. doi: 10.1111/j.1471-6402.1997.tb00108.x

Gardner, C. B. (1995). Passing by: Gender and public harassment. Berkeley, CA: University of California Press.

Gelfand, M. J., Fitzgerald, L. F., \& Drasgow, F. (1995). The structure of sexual harassment: A confirmatory analysis across cultures and settings. Journal of Vocational Behavior, 47, 164-177. doi: 10.1006/jvbe.1995.1033 
Goffman, E. (1963). Behavior in public places: Notes on the social organization of gatherings. New York, NY: Free Press of Glencoe.

Golden, J. H., III, Johnson, C. A., \& Lopez, R. A. (2001). Sexual harassment in the workplace: Exploring the effects of attractiveness on perception of harassment. Sex Roles, 45, 767-784.

Grossman, A. J. (2008, May 14). Catcalling: Creepy or a compliment? CNN.com. Retrieved from http://www.cnn.com/2008/LIVING/personal/05/14/lw.catcalls/in dex.html?iref=allsearch

Hallstein, L. D. O. (1999). A postmodern caring: Feminist standpoint theories, revisioned caring, and communication ethics. Western Journal of Communication, 63, 32-57. doi: 10.1080/10570319909374627

Harding, S. G. (1986). The science question in feminism. Ithaca, NY: Cornell University Press.

Hartsock, N. C. M. (1983) Money, sex, and power: Toward a feminist historical materialism. New York, NY: Longman.

Heben, T. (1994). A radical reshaping of the law: Interpreting and remedying street harassment. South California's Review of Law and Women's Studies, 4, 183-219.

Hickson, M., III, Grierson, R. D., \& Linder, B. C. (1990). A communication model of sexual harassment. Association for Communication Administration Bulletin, 74, 22-33.

Hornstein, E. (1984). Sexual harassment. In The Guide to American Law: Everyone's Legal Encyclopedia (pp. 233-235). St. Paul, MN: West Publishing.

Hunt, C. J., \& Gonsalkorale, K. (2014). Who cares what she thinks, what does he say? Links between masculinity, in-group bonding and gender harassment. Sex Roles, 70, 14-27. doi: 10.1007/s11199-013-0324-х

Kearl, H. (2010). Stop street harassment: Making public places safe and welcoming for women. Santa Barbara, CA: Praeger.

Keyton, J., Ferguson, P., \& Rhodes, S. C. (2001). Cultural indicators of sexual harassment. Southern Journal of Communication, 67, 33-50. doi: $10.1080 / 10417940109373217$

Kissling, E. A. (1991). Street harassment: The language of sexual terrorism. Discourse \& Society, 2, 451-460. doi: 10.1177/0957926591002004006 
Kissling, E. A., \& Kramarae, C. (1991). Stranger compliments: The interpretation of street remarks. Women's Studies in Communication, 14, 75-93. doi: 10.1080/07491409.1991.11089751

Lahsaeizadeh, A., \& Yousefinejad, E. (2012). Social aspects of women's experiences of sexual harassment in public places in Iran. Sexuality \& Culture, 16, 17-37. doi: 10.1007/s12119-011-9097-y

Lambertz, K. A. (2014, October 7). Woman shot, killed after saying no to a man's advances, Detroit police say. Huffingtonpost.com. Retrieved from http://www.huffingtonpost.com /2014/10/07/mary-spears-killeddetroit_n_5945518.html

Laniya, O. O. (2005). Street smut: Gender, media, and the legal power dynamics of street harassment, or 'hey sexy' and other verbal ejaculations. Columbia Journal of Gender and Law, 14, 91-130.

Levine, M. (1999). Rethinking bystander nonintervention: Social categorization and the evidence of witnesses at the James Bulger murder trial. Human Relations, 52, 1133-1155. doi: 10.1177/001872679905200902

Leonardo, M. D. (Summer, 1981). Political economy of street harassment. Aegis, 51-57. Retrieved from http://www.stopstreetharassment.org/

Lincoln, Y. S., \& Guba, E. G. (1985). Naturalistic inquiry. Beverly Hills, CA: SAGE.

Lindlof, T. R., \& Taylor, B. C. (2011). Qualitative communication research methods $\left(3^{\text {rd }}\right.$ ed.). Thousand Oaks, CA: SAGE.

Lyman, P. (1987). The fraternal bonds as a joking relationship. A case study of the role of sexist jokes in male group bonding. In M. S. Kimmel (Ed.), Changing men: New directions in research on men and masculinity (pp. 148-153). Thousand Oaks, CA: SAGE.

Lord, T. (2009). The relationship of gender-based public harassment to body image, self-esteem, and avoidance behavior (Doctoral dissertation). Retrieved from ProQuest Dissertations \& Theses Full Text. (Order No. 3369986).

MacKinnon, C. A. (1979). Sexual harassment of working women: A case of sex discrimination. New Haven, CT: Yale University Press. 
Macmillan, R., Nierobisz, A., \& Welsh, S. (2000). Experiencing the streets: Harassment and perceptions of safety among women. Journal of Research in Crime \& Delinquency, 37, 306-322. doi: 10.1177/0022 427800037003003

Madriz, E. I. (1997). Images of criminals and victims: A study on women's fear and social control. Gender and Society, 11, 342-356. doi: 10.1177/089124 397011003005

McCarty, M., Iannone, N., \& Kelly, J. (2014). Stranger danger: The role of perpetrator and context in moderating reactions to sexual harassment. Sexuality \& Culture, 18, 739-758. doi:10.1007/s12119-013-9215-0

Megarry, J. (2014). Online incivility or sexual harassment? Conceptualising women's experiences in the digital age. Women's Studies International Forum, 47, 46-55. doi:10.1016/j.wsif.2014.07.012

Metts, S., \& Cupach, W. R. (2008). Face theory. In L.A. Baxter, \& D.O. Braithwaite (Eds.), Engaging theories in interpersonal communication (pp. 203-214). Newbury Park, CA: Sage.

Metts, S., \& Planalp, S. (2011). Emotion experience and expression: Current trends and future directions in interpersonal relationship research. In M. Knapp \& J. Daly (Eds.), Handbook of interpersonal communication (4th ed., pp. 283-315). Newbury Park, CA: Sage.

Nielsen, L. B. (2000). Situating legal consciousness: Experiences and attitudes of ordinary citizens about law and street harassment. Law and Society Review, 34, 1055-1090. doi: 10.2307/3115131

O'Neil, F.M. (2013). Gender in public space: Policy frameworks and the failure to prevent street harassment (Master's thesis). Retrieved from http://www.stopstreet harassment.org /2013/07/jarrahthesis/

Postmes, T., \& Spears, R. (1998). Deindividuation and antinormative behavior: A metaanalysis. Psychological Bulletin, 123(3), 238. doi: 10.1037//00332909.123.3.238

Pryor, J. B. (1998). The Likelihood to Sexually Harass Scale. In C. M. Davis, W. H. Yarber, R. Bauserman, G. Schreer, \& S. L. Davis (Eds.), Sexuality-related measures: A compendium (pp. 295-298). Beverly Hills: SAGE.

Pryor, J. B., LaVite, C. M., \& Stoller, L. M. (1993). A social psychological analysis of sexual harassment: The person/situation interaction. Journal of Vocational Behavior, 42, 68-83. doi: 10.1006/jvbe.1993.1005 
Pryor, J. B., \& Meyers, A. B. (2000). Men who sexually harass women. In L. B.

Schlesinger (Ed.), Serial Offenders: Current thought, recent findings (pp. 207228). Boca Raton: CRC Press.

Pryor, J. B., \& Stoller, L. M. (1994). Sexual cognition processes in men high in the likelihood to sexually harass. Personality and Social Psychology Bulletin, 20, 163-169. doi: 10.1177/0146167294202003

Rakow, L. F., \& Wackwitz, L. A. (2004). Feminist communication theory: Selections in context. Thousand Oaks, CA: SAGE.

Rawlins, R. (2012). "Whether I'm an American or not, I'm not here so you can hit on me": Public harassment in the experience of U.S. women studying abroad. Women's Studies, 41, 476-497. doi:10.1080/00497878.2012.663263

Rotundo, M., Nguyen, D., \& Sackett, P. R. (2001). A meta-analytic review of gender differences in perceptions of sexual harassment. Journal of Applied Psychology, 86, 914-922. doi:10.1037//0021-9010.86.5.914

Sheffield, C. J. (1987). Sexual terrorism: The social control of women. In M.M. Feree, \& B.B Hess (Eds.), Analyzing gender (pp. 171-189). Newbury Park, CA: SAGE.

Sheffield, C. J. (1989). The invisible intruder: Women's experiences of obscene phone calls. Gender and Society, 3, 483-488. doi: 10.1177/089124389003004006

Spitzberg, B. H. (1998). Sexual coercion in courtship relations. In B. H. Spitzberg \& W. R. Cupach (Eds.), The dark side of close relationships (pp. 179-232). Mahwah, N.J.: Lawrence Erlbaum Associates.

Stanko, E. (1985). Intimate intrusions: Women's experience of male violence. London: Routledge.

Street harassment of women and girls in New York City: Oversight hearing before New York City Council's Committee on Women's Issues (2010).

Stopstreetharassment.org. (Testimony of S. Song). Retrieved from http://www.stopstreetharassment.org/toolkits/nyccouncil hearing2010/

Thomae, M., \& Pina, A. (2015). Sexist humor and social identity: The role of sexist humor in men's in-group cohesion, sexual harassment, rape proclivity, and victim blame. Humor: International Journal of Humor Research, 28, 187-204. doi:10.1515/humor-2015-0023 
Thompson, D. M. (1994). Woman in the street: Reclaiming the public space from sexual harassment. The Yale Journal of Law \& Feminism, 6, 313-348. Retrieved from http://www.heinonline.org.libproxy.lib.ilstu.edu/HOL/Page?page=313\&handle= hein.journals $\% 2$ Fyjfem6\&collection=journals

Tran, M. (2015). Combatting gender privilege and recognizing a woman's right to privacy in public spaces: Arguments to criminalize catcalling and creepshots. Hastings Women's Law Journal, 26, 185-206. Retrieved from http://www.lexisnexis.com.libproxy.lib.ilstu.edu/hottopics/lnacademic/?verb=sr $\& \mathrm{csi}=172728$

Tuerkheimer, D. (1997). Street harassment as sexual subordination: The phenomenology of gender-specific harm. Wisconsin Women's Law Journal, 12, 167-206.

Wesselmann, E., \& Kelly, J. (2010). Cat-calls and culpability: Investigating the frequency and functions of stranger harassment. Sex Roles, 63, 451-462. doi: $10.1007 / \mathrm{s} 11199-010-9830-2$

White, C. H. (2015). Expectancy violations theory and interaction adaptation theory: From expectations to adaptation. In D. O. Braithwaite \& P. Schrodt (Eds.), Engaging theories in interpersonal communication: Multiple perspectives $\left(2^{\text {nd }}\right.$ ed.) (pp. 1-20). Thousand Oaks, CA: SAGE.

Wood, J. T. (1994). Saying it makes it so: The discursive construction of sexual harassment. In S. G. Bingham (Ed.), Conceptualizing sexual harassment as discursive practice (pp. 17-44). Westport, CT: Praeger.

Wood, J. (2008). Critical feminist theories: Giving voice and visibility to women's experiences in interpersonal communication. In L. Baxter \& D. O. Braithwaite (Eds.), Engaging theories in interpersonal communication: Multiple perspectives (pp. 323-335). Thousand Oaks, CA: SAGE.

Young, I. M. (2005). On female body experience: "Throwing like a girl" and other essays. New York, NY: Oxford University Press. 


\section{APPENDIX A}

\section{EMAIL MESSAGE/FACEBOOK MESSAGE}

\section{TO PROSPECTIVE PARTICIPANTS}

\section{Dear Prospective Participant,}

As a graduate student in the School of Communication at Illinois State University, I am conducting a qualitative study about women's experiences with men making comments towards them in public places - that is, how they conceptualize these behaviors - under the guidance of Dr. John Baldwin as part of my Master's Thesis. I am interested in the standpoints of women who have experienced men's comments in public spaces. I would greatly appreciate your participation in this study. Your participation would be in the form of a one-on-one confidential interview.

To participate, you must be over the age of 18 , female, and have experienced men making comments towards you in public places. If you choose to participate in this study, please contact Colleen using the information provided below to set up a meeting location and time. This interview is expected to last between 30-60 minutes. This interview will ask you to respond to a series of questions relating to your experiences with men's comments and your emotional responses to the experiences. The interview will be audio recorded on the interviewer's laptop, which is password protected; however, we can do the interview without audio recording if that is your preference.

Your participation is completely voluntary, and anything you answer during the interview will remain completely confidential. There is no penalty for choosing not to participate in this study. Furthermore, you have the right to decline to answer any question or withdraw from the study at any time without penalty or adversely affecting your relationship with Colleen, Dr. Baldwin, or Illinois State University.

To move forward with participating in this study, please contact Colleen O'Leary by email at coleary@ IllinoisState.edu.

Please direct any questions and/or comments to Dr. John Baldwin (jrbaldw@IllinoisState.edu) or to us.

Thank you for your time and consideration. 


\section{APPENDIX B}

\section{INFORMED CONSENT}

I am a graduate student under the direction of Dr. John Baldwin in the School of Communication at Illinois State University. I am inviting you to participate in a research study to understand women's standpoints on men's comments towards them in public places as part of a Master's Thesis.

Your participation-will involve an interview regarding your experiences of men making comments towards you, especially your emotional and communicative responses to such experiences. The interview should take between 30-60 minutes. It will be audio recorded on a tape recorder and transferred to the interviewer's personal lap top, which is password protected, and your audio file will not contain any identifying marks. The audio recording will be destroyed once the transcription and analysis are complete. Still, you may feel uncomfortable being audio recorded; if you do, we can proceed with the interview without the audio recording, and the researcher will summarize the interview from her memory after the session.

Your participation in this study is voluntary. If you choose not to participate or to withdraw from the study at any time, there will be no penalty (it will not affect your relationship with Colleen O'Leary, Dr. John Baldwin, or Illinois State University). You can refuse to answer any question you do not wish to answer. Your responses are completely confidential. To ensure your confidentiality, we will not reveal any anything else that identifies any participant. Please be aware that if you have chosen a public location for the interview, we cannot guarantee that anyone who overhears our conversation will maintain confidentiality.

We believe there are minimal risks associated with participating in this project. You may feel some discomfort discussing your experiences. If you would like to see a professional to discuss any discomfort, you may, if you are a student at ISU, contact ISU Student Counseling Services at (309) 438-3655. If you are not an ISU student, you may, at your expense, contact a counselor of your choice. You will receive no compensation for this study. Your participation will help extend our understanding of women's experiences of and responses to men's comments in public places. 
Your signature below indicates that you are voluntarily making a decision to participate in this research study and have read the information presented above. You will be given a copy of this consent form to keep. Please direct any questions and/or comments to Dr. John Baldwin (jrbaldw@ilstu.edu) or to me (coleary@ilstu.edu). For questions regarding your rights as a research participant you are encouraged to contact the Illinois State University Research Ethics and Compliance Office: Phone: 309-438-2529, Email: rec@ilstu.edu.

Thank you for your assistance.

Colleen O'Leary

Graduate Student

Illinois State University, School of Communication

coleary@ilstu.edu

Please Print Name

I consent to participate in this research: $\square$ Yes $\quad \square$ No

Please Sign Name

Date

I consent for the interview to be audio recorded: $\square$ Yes $\quad \square$ No

Please Sign Name

Date 


\section{APPENDIX C \\ INTERVIEW GUIDE/QUESTION PROTOCOL}

\section{Demographic Questions}

1. Are you ready to begin?

2. Is it okay with you that I audio record this interview?

3. How old are you?

4. Do you currently attend Illinois State University?

5. What year in school are you?

6. Are you currently in a long-term committed relationship?/What is your current romantic relationship status?

Transition: Now I am going to start by asking about your experiences with a male stranger making a comment to you in a public space. Please keep in mind that that this section of the interview is focused on the discussion of previous experiences. Try to think back upon these questions and respond to the best of your knowledge.

\section{Experiences with Catcalling Questions}

1. Have you ever been the recipient of a comment from a male stranger in a public place?

a. Tell me about that experience

b. How did you feel during/after that experience?

c. What emotions did you feel at that time?

2. Describe what the person was like

a. Anything else you can remember?

3. What was your response?

a. Would you have responded differently thinking back to that experience?

4. Did you communicate your experience with your friends/family/others?

a. Why did/didn't you communicate with others about the incident?

b. What was their reaction?

c. Did their reaction to the incident change how you felt about it?

5. Did the context affect how you perceived the comment?

a. Was the stranger in a group or by themselves?

b. Did you feel safe in that location before the incident occurred? After?

c. Did what the stranger was doing at that time affect what you were doing? 
6. Have you experienced a stranger's comments any other times?

a. Tell me about that/those experience(s)?

b. How did this/those experience(s) differ from the one you talked about earlier?

c. Can you give me another example of a stranger making a comment to you in a public place?

7. How would you define a catcall?

a. Would you consider the experiences you discussed previously as catcalling?

b. Do you think catcalling is a positive/negative/combination experience?

Transition: In other cultures, there tends to be two predominant views of catcalling: the comments are complimentary and the comments are rude or offensive. These ideologies seem to be at odds with one another in terms of how catcalling is interpreted. For these next questions, think about your perceptions of catcalling as well as your efforts in trying to make sense of catcalling behaviors.

\section{Perceptions of Catcalling Questions}

1. Describe an instance in which you would consider a catcall a compliment.

a. What verbal remarks would be involved?

b. Why would you consider the remarks complimentary?

c. Is there something that influenced you to view catcalling in this way?

Definition of catcalling: the "use of crude language, verbal expression, and nonverbal expression that takes place in public areas such as streets, sidewalks, or bus stops" (p. 276).

2. Describe an instance in which you would consider a catcall to be rude or offensive.

a. What verbal remarks would be involved?

b. Why would you consider the remarks to be rude or offensive?

c. Is there something that influenced you to view catcalling in this way?

3. Do certain characteristics of the catcaller matter when identifying the remarks as complimentary or rude?

a. What are the characteristics?

b. Why do certain characteristics matter?

4. How does the context in which catcalling occurs affect your perceptions?

a. Why do you think this is?

5. Are there occasions or circumstances that might make a catcall more or less threatening? 
a. Would you view a catcall as more or less threatening if you were with a group of friends and the catcaller was alone?

b. Why would you view a catcall in that way?

c. Would you view a catcall as more or less threatening if the catcaller was with a group of friends and you were alone?

d. Why would you view a catcall in that way?

6. If you were the recipient of a catcall in the near future how would you respond?

a. Why would you respond in that manner?

7. Do you do anything in your everyday life to avoid being the recipient of a catcall?

8. Some people have classified catcalling as a form of harassment, what is your response to that?

Closing question: Is there anything else that you would like to say about any of the things that we have discussed? Have you ever known any women who have catcalled? 
APPENDIX D

PARTICIPANT INFORMATION

\begin{tabular}{|c|c|c|c|}
\hline Participant & Age & Race & Relationship Status \\
\hline Mary & 19 & Caucasian & Single \\
\hline Maddie & 21 & Caucasian & Single \\
\hline Leslie & 25 & Unknown & $\begin{array}{l}\text { Long-term } \\
\text { committed } \\
\text { relationship }\end{array}$ \\
\hline Lindsey & 22 & Caucasian & Single \\
\hline Blaire & 25 & Caucasian & Single \\
\hline Gina & 24 & Unknown & Engaged \\
\hline Halle & 23 & Caucasian & $\begin{array}{l}\text { Long-term } \\
\text { committed } \\
\text { relationship }\end{array}$ \\
\hline Paige & 24 & Unknown & $\begin{array}{l}\text { Long-term } \\
\text { committed } \\
\text { relationship }\end{array}$ \\
\hline Riley & 22 & Caucasian & Single \\
\hline Diana & 21 & Caucasian & Single \\
\hline Allie & 24 & Caucasian & Single \\
\hline Ella & 19 & Caucasian & $\begin{array}{l}\text { Long-term } \\
\text { committed } \\
\text { relationship }\end{array}$ \\
\hline Katie & 26 & Caucasian & Engaged \\
\hline
\end{tabular}

